\title{
HEAVY ION FUSION SCIENCE VIRTUAL NATIONAL LABORATORY 4th QUARTER 2008 MILESTONE REPORT
}

\author{
Carry out initial target experiment in the new target \\ chamber, using beams compressed and focused by an \\ improved bunching waveform and a final focus solenoid.
}

F.M. Bieniosek, A. Anders, J.J. Barnard, M.R. Dickinson, W. Greenway, E. Henestroza, T. Katayanagi, B.G. Logan, C.W. Lee, M. Leitner, S. Lidia, R. M. More, P. Ni, P. K. Roy, P. A. Seidl, W. Waldron LBNL

Accelerator Fusion Research Division Ernest Orlando Lawrence Berkeley National Laboratory

University of California

Berkeley, California 94720

September 2008

This work was supported by the Director, Office of Science, Office of Fusion Energy Sciences, of the U.S. Department of Energy under Contract No. DE-AC02-05CH11231. 


\title{
HEAVY ION FUSION SCIENCE VIRTUAL NATIONAL LABORATORY
}

\author{
4th QUARTER 2008 \\ MILESTONE REPORT
}

Carry out initial target experiment in the new target chamber, using beams compressed and focused by an improved bunching waveform and a final focus solenoid.

F.M. Bieniosek, A. Anders, J.J. Barnard, M.R. Dickinson, W. Greenway, E. Henestroza, T. Katayanagi, B.G. Logan, C.W. Lee, M. Leitner, S. Lidia, R. M. More, P. Ni, P. K. Roy, P. A. Seidl, W. Waldron

for the HIFS-VNL

September 16, 2008 


\section{SUMMARY}

This milestone has been met. In the previous quarter ( $3^{\text {rd }}$ quarter FY2008), the Heavy Ion Fusion Science Virtual National Laboratory (HIFS-VNL) completed the new experimental target chamber facility for future Warm Dense Matter (WDM) experiments [1]. The target chamber is operational and target experiments are now underway, using beams focused by a final focus solenoid and compressed by an improved bunching waveform. Initial experiments have demonstrated the capability of the Neutralized Drift Compression Experiment (NDCX) beam to heat bulk matter in target foils. The experiments have focused on tuning and characterizing the NDCX beam in the target chamber, implementing the target assembly, and implementing target diagnostics in the target chamber environment.

We have completed a characterization and initial optimization of the compressed and uncompressed NDCX beam entering the target chamber. The neutralizing plasma has been significantly improved to increase the beam neutralization in the target chamber.

Preliminary results from recent beam tests of a gold cone for concentrating beam energy on target are encouraging and indicate the potential to double beam intensity on target. Other advantages of the cone include the large amount of neutralizing secondary electrons expected from the grazing incidence at the cone walls, and the shielding of the target from the edges of the beam pulse.

The first target temperature measurements with the fast optical pyrometer were made on Sep. 12, 2008. The fast optical pyrometer is a unique and significant new diagnostic. These new results demonstrate for the first time beam heating of the target to a temperature well over $2000 \mathrm{~K}$.

The initial experimental results are suggestive of potentially interesting physics. The rapid initial rise and subsequent decay of the target temperature during the beam pulse indicate changes in the balance of beam heating and target evaporative cooling, a behavior which may be affected by phenomena such as droplet formation and rapid changes in the optical properties of the hot target material. NDCX, possibly uniquely, is capable of studying these changes because of its wide range of diagnostic capabilities. These capabilities include target diagnostics already in place such as the fast pyrometer and streak camera, as well as the ability to measure both ion beam transmission and optical transmission through the foil. Measurements with these diagnostic techniques can help determine the rate at which the target is breaking up into droplets and the rate at which its bulk optical properties are changing. 


\section{INTRODUCTION}

The US heavy ion fusion science program is developing techniques for heating ion-beam-driven warm dense matter (WDM) targets [1-4]. Intense ion beams have several attractive features as a technique for generating WDM. These features include:

-Precise control of local beam energy deposition $\mathrm{dE} / \mathrm{dx}$, nearly uniform throughout a given volume, and not strongly affected by target temperature,

-Large sample sizes (about 1 micron thick by $1 \mathrm{~mm}$ diameter),

-The ability to heat any target material, for example, foams, powders, conductors, insulators, solid, gas, etc.

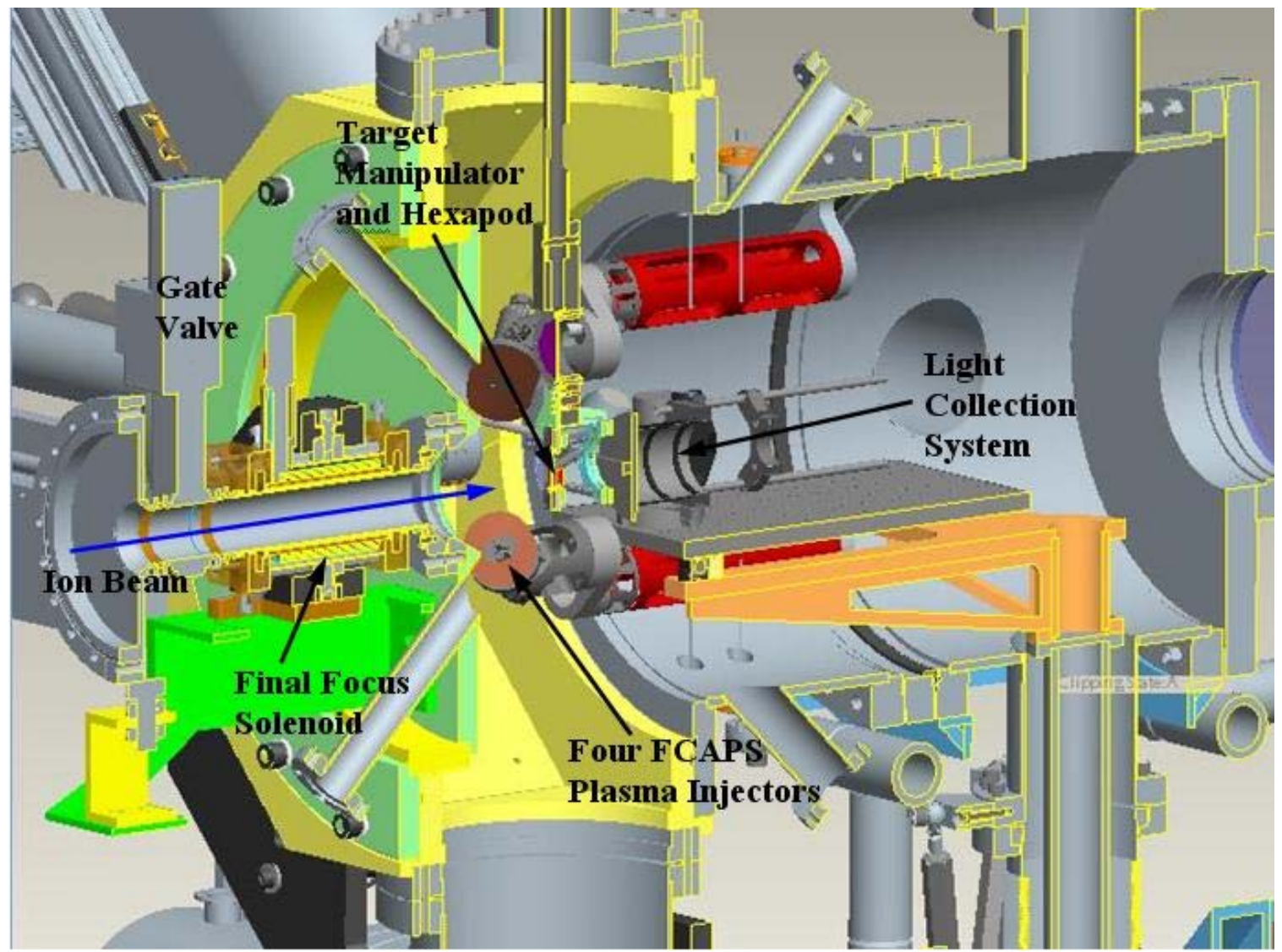

Figure 1: 3D CAD view of the target chamber. The target foil is mounted in the center of the chamber.

A detailed description of the new experimental target chamber, targets, and target diagnostics was reported in the 3rd Quarter 2008 milestone report [1]. The target chamber provides:

1. A dense $\left(10^{12}\right.$ to $\left.10^{14} \mathrm{~cm}^{-2}\right)$ plasma injection to neutralize the space charge of the incoming ion beam,

2. a strong (8 Tesla) final focus solenoid for radial ion beam compression,

3 . vacuum pumping, 
4. retractable ion beam diagnostics,

5. a retractable target holder with in-situ alignment capabilities,

6. fast optical target diagnostics with access to the front, side and back of the target foil,

7. accurate (micron range) alignment capabilities.

The WDM target diagnostics include a high-speed multi-channel optical pyrometer, optical streak camera, VISAR, and high-speed gated cameras [1,5]. The fast optical pyrometer is a unique and significant new diagnostic which provides valuable information on the temperature evolution of the heated target [6]. Figure 1 displays a schematic view of the new target chamber facility, which is installed downstream of the induction bunching module on NDCX-1. The longitudinally compressing ion beam enters from the left and is focused to $\sim 1 \mathrm{~mm}$ spot size by the final focus solenoid. 


\section{NDCX BEAM CHARACTERIZATION}

\subsection{Changes to NDCX beamline}

The NDCX experiment has been updated and improved in several ways to increase the fluence of the ion beam onto the target and to increase the resolution and sensitivity of the diagnostic package. The NDCX beamline is shown in Fig. 2.

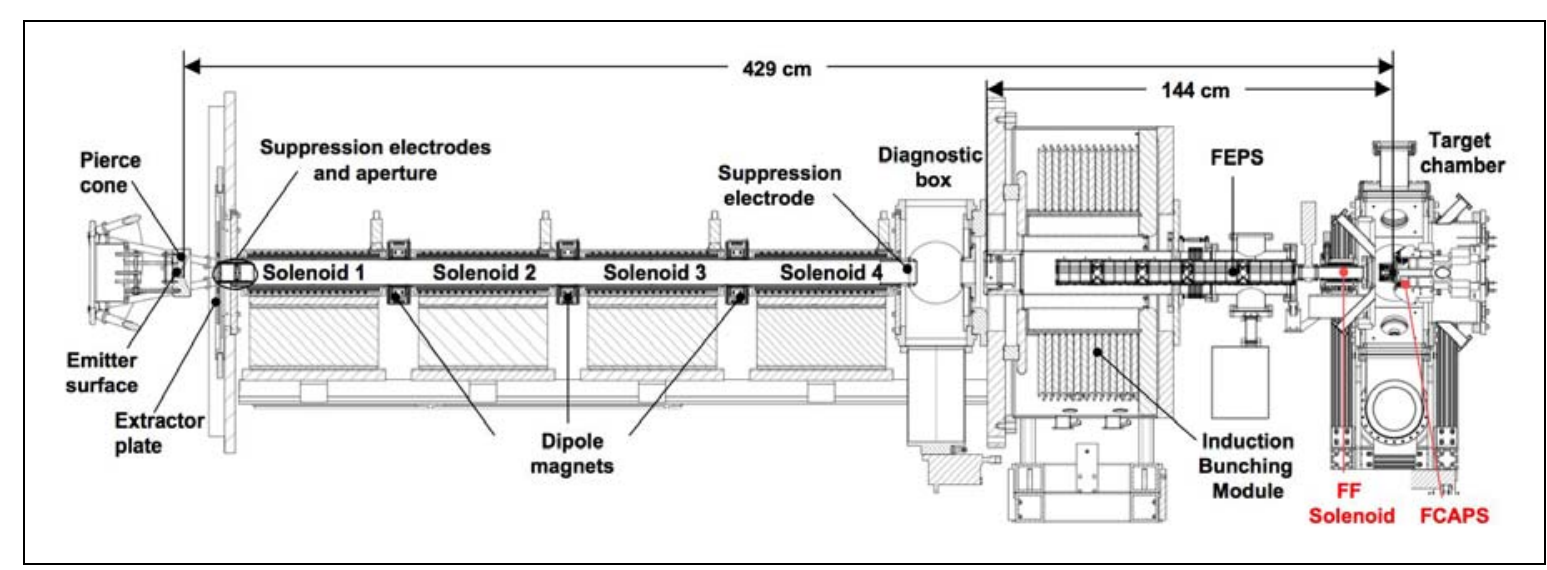

Fig. 2 : NDCX beamline.

The emitter has been replaced after several years of service. The new $\mathrm{K}^{+}$emitter reliably produces $30-38 \mathrm{~mA}$ for $300-350 \mathrm{kV}$ diode extraction voltages after aperturing and prior to transport downstream. Phase space measurements show excellent space charge dominated beam distributions with 4-rms emittances of $\sim 17 \mathrm{~mm}$-mrad.

A new 8-Tesla pulsed Final Focus Solenoid (FFS) has been fabricated and installed at the target chamber entrance, and replaces the previous solenoid that produced a 5-Tesla peak field. (The peak field of the first solenoid was limited by high-voltage flashover near the magnet leads. The design of the new solenoid was improved, allowing consistent operation up to the design value of 8 Tesla.) The physical dimensions of the new solenoid resulted in a $0.13-\mathrm{cm}$ increase in the beamline length between the Induction Bunching Module (IBM) and the target plane. This shift is sufficient to require retuning of the IBM waveform and upstream matching optics.

The plasma density in the target chamber has been increased and now mostly exceeds the on-axis beam density to satisfy the condition $Z_{p} / n_{b}>1$, where $n_{p}$ and $n_{b}$ are the plasma and ion beam density, respectively, and $Z$ is the mean ion charge state of the plasma ions. This work consists of several segments. We first measured the plasma density of two cathodic arc plasma sources (two-FCAPS) by an axially driven Langmuir probe with and without a 4-Tesla solenoid magnetic field. Next, the two-FCAPS system was replaced by fabricating four cathodic arc plasma sources (four-FCAPS) with straight filters (vs $45^{\circ}$ ) to increase the plasma density. The solenoid magnetic field was increased to 8T. Results show that the new four-FCAPS system with the short straight filters provides 9-60 times greater plasma density than the two-FCAPS system with the bent filters. Assuming a plasma velocity of $2 \times 10^{4} \mathrm{~m} / \mathrm{sec}$, the four-FCAPS system provided a peak plasma density $9 \times 10^{12}-6 \times 10^{13} / \mathrm{cm}^{3}$. Finally, the axial and radial plasma 
distribution was measured with a specially designed array of multiple 37 collectors. This new probe data shows that plasma forms a thin column of diameter $\sim 5 \mathrm{~mm}$ along the solenoid axis when the FFS is energized. The last two segments are described below.

\section{Axial plasma density of four-FCAPS system}

Figures 3(a) and 3(b) show the four new plasma sources. The diameter of each of the aluminum cathodes is $0.64 \mathrm{~cm}$ [Fig. 3(c)]. Each of the four CAPS makes an angle of $50^{\circ}$ with the beam axis. The filters, which also serve as the arc discharge anode, are shorter and straight compared to the previous two FCAPS. The distance between a cathode surface to the first and last turns of the filter are 0.64 and $3.57 \mathrm{~cm}$, respectively. The distance between the center of a cathode to the ion beam axis is $9.3 \mathrm{~cm}$. The new CAPS system was installed in the target chamber [Fig. 3(d)] along with the Langmuir probe and the FFS to measure the plasma density as a function of location. In this new configuration, the distance between the mid-plane of the FFS and the target plane was $19.3 \mathrm{~cm}$. All four CAPS were connected to a pulse forming network (PFN) capable of providing $\sim 800 \mathrm{~A}$ per plasma source.
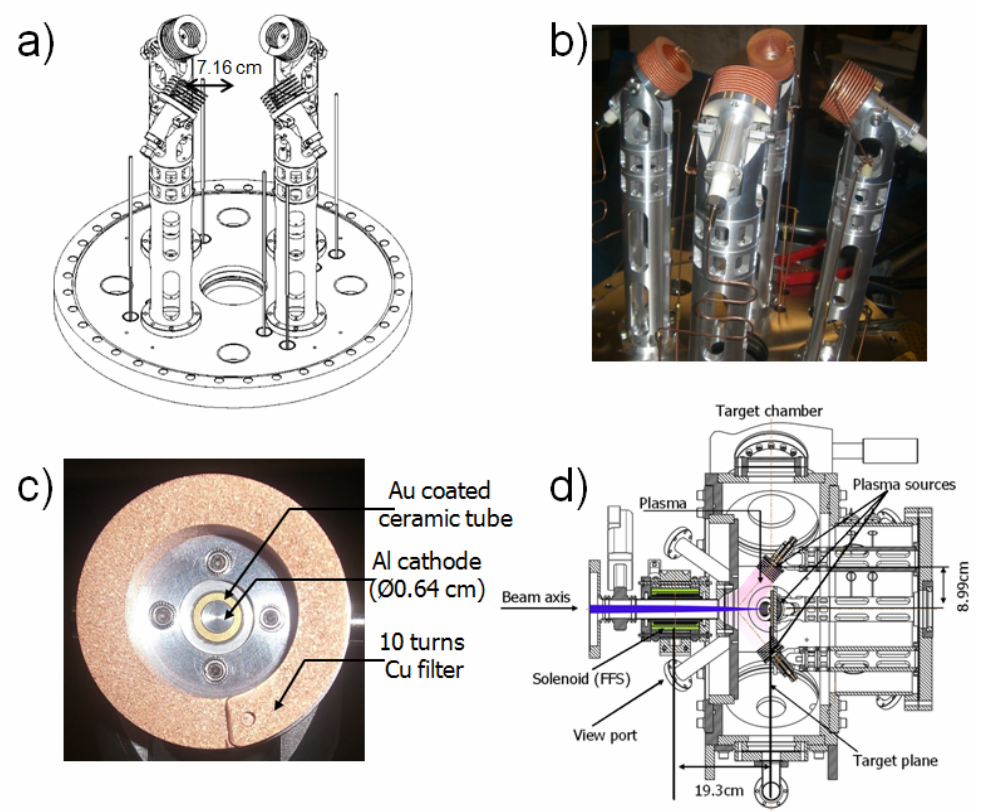

Fig. 3. (a) Schematic of the four filtered cathodic arc plasma sources; (b) photograph of the CAPS; (c) photograph of the cathode and filter; and (d) a sketch of the target chamber with four cathodic are plasma sources and the final focus solenoid (FFS).

Figure 4 shows the plasma profile of the four-FCAPS. The cross sectional area of the Langmuir probe tip is $1.46 \mathrm{~mm}^{2}$. The axial plasma density distribution in the solenoid and between the solenoid and the NDCX target plane was measured, for magnetic field $\mathrm{B}=8 \mathrm{~T}(21 \mathrm{kA}$ peak current) and $\mathrm{B}=0$. Each of the four CAPS was operated with a discharge current of 770 Amperes. Results show that the new four-CAPS system with the short straight filters provided 9-60 times greater plasma density than the two-FCAPS system with the bent filters. Assuming a plasma velocity of $2 \times 10^{4} \mathrm{~m} / \mathrm{sec}$, the four-FCAPS system provided a plasma density $\approx 10^{13} / \mathrm{cm}^{3}$. 
a)
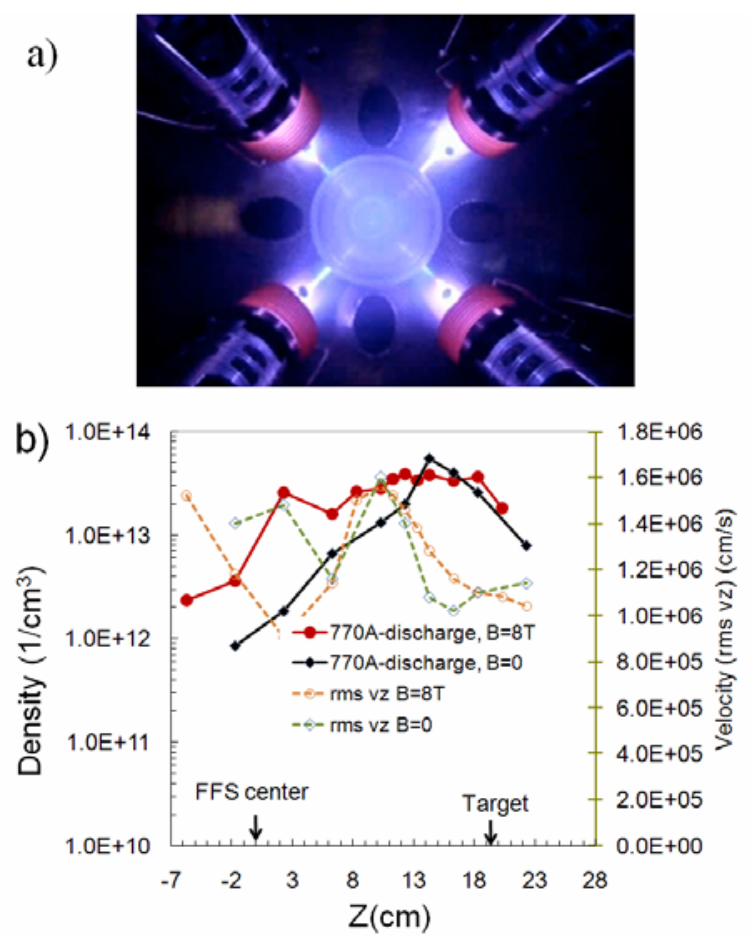

Fig.4. (a) Discharge of the four-CAPS, captured with a video camera; and (b) axial plasma density and rms velocity.

\section{Axial and radial plasma measurement by a probe of multiple arrays of collectors}

A new radial plasma probe, Fig. 5(a) was developed and axial and radial plasma density was measured. Details of the probe geometry are described elsewhere [7]. Figure 5(b) shows the plasma density at the center of the beam axis, measured using the center collector of the probe, when the FFS was on and off. A plasma density of $\sim 1.1 \times 10^{13} \mathrm{~cm}^{-3}$ was measured at the entrance to the FFS, and this density dropped to $\approx 10^{12} \mathrm{~cm}^{-3}$ between the FFS magnet and the target plane when the FFS magnet was operated with $8 \mathrm{~T}$. The highest density was on the beam axis, within a diameter of $\sim 5 \mathrm{~mm}$, in the final focus solenoid when the solenoid was powered. Figure 6 shows surface plots of the measured transverse and axial plasma distribution using the 37 collectors of the radial probe. The FFS was powered (8T), and the probe was driven from the upstream end of the FFS to the target plane. The maximum plasma density at the center of the FFS was $\approx 1 \times 10^{13}$ $\mathrm{cm}^{-3}$ for the measured plasma current of $53 \mathrm{~mA}$ for $\mathrm{Al}^{2+}$, assuming a velocity from LSP modeling of $1.5 \times 10^{4} \mathrm{~m} / \mathrm{sec}$. Figure 7 shows 3-D electrostatic PIC simulation, using the Warp code, of the four CAPS plasma density when $\mathrm{B}=8 \mathrm{~T}$. In this simulation $\mathrm{Al}^{+}$plasma, with velocity of $3 \mathrm{~cm} / \mu \mathrm{s}$, and temperature of $7 \mathrm{eV}$ was assumed. Simulation results are shown at 4.5 $\mu \mathrm{s}$ after the beginning of plasma injection, and show qualitative agreement with the experiment. The density on axis shows similar double-humped profile, with a peak inside the solenoid. The density rapidly falls off axis inside the solenoid. Note that the peak density is only one tenth of the experimentally determined value. This is possibly due to underestimation of the injected plasma density coming from the four-CAPS, and an overall normalization uncertainty of the Langmuir probe data. 

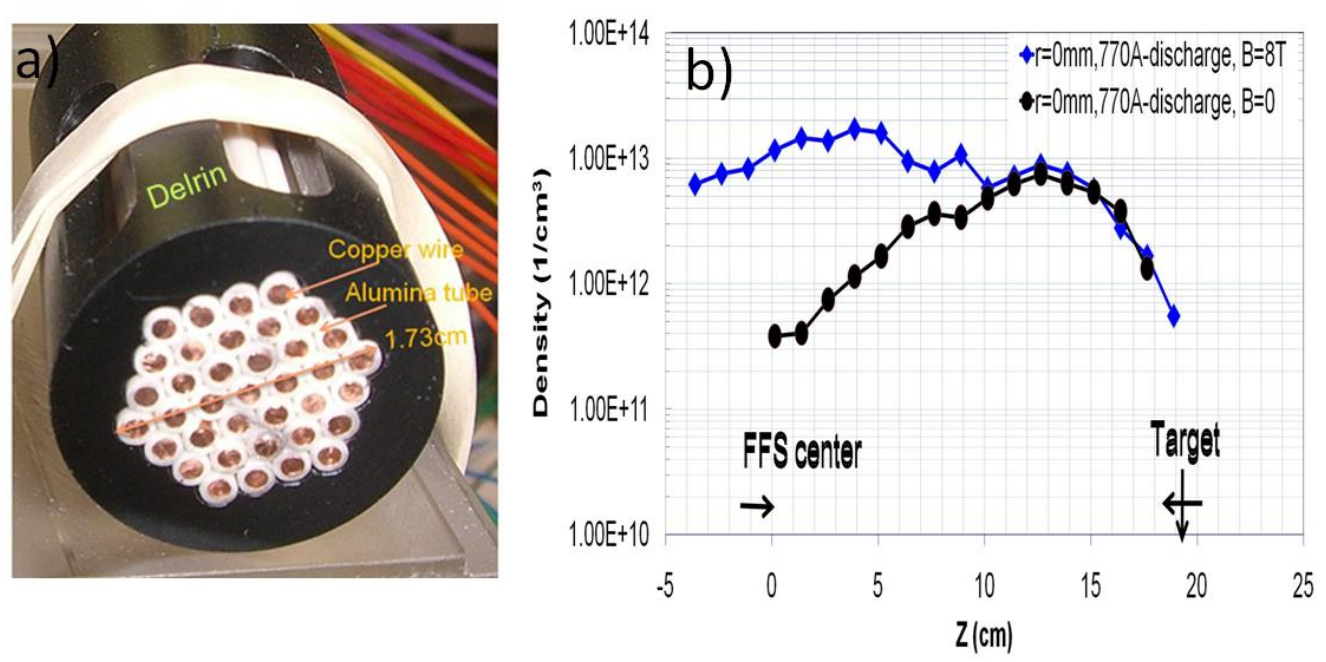

Fig. 5 (a) 37-collector probe array. Each probe is $1.6 \mathrm{~mm}$ in diameter, with a collective diameter of $1.73 \mathrm{~cm}$. (b) Axial plasma density measured using only the center collector, when the FFS was powered (line with solid diamonds) and not powered (line with solid circles).
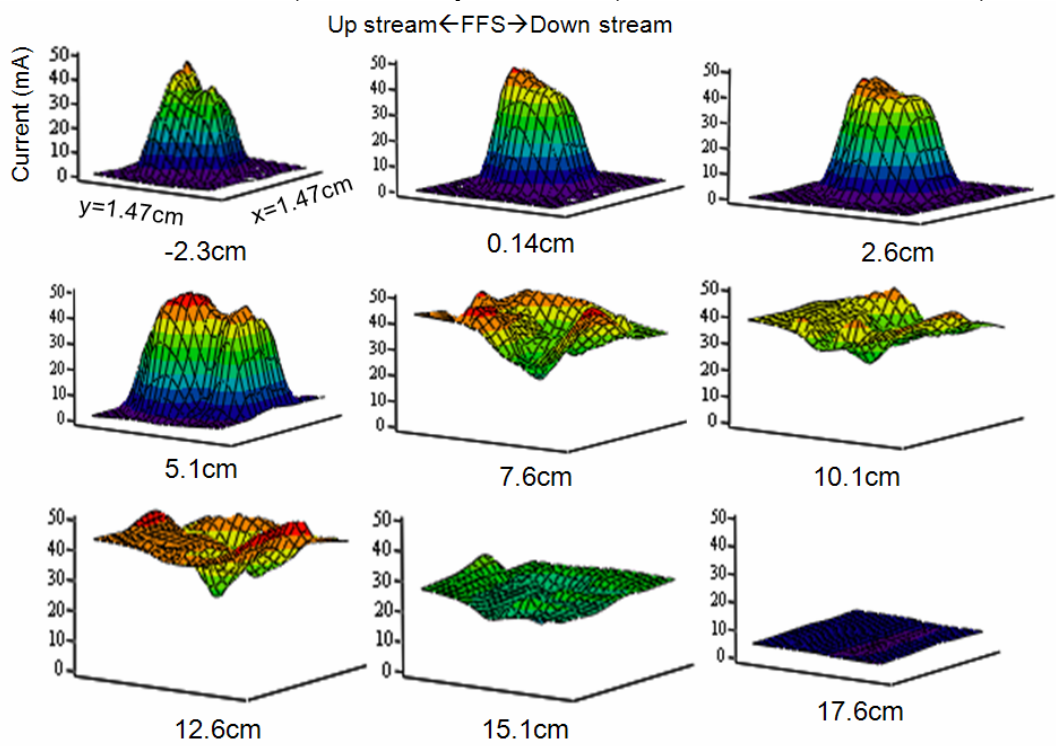

Fig.6. Surface plots of the measured (using the 37 collectors) transverse and axial plasma distribution when the FFS and the four-CAPS were operated with $8 \mathrm{~T}$ and $770 \mathrm{~A}$, respectively. The probe was driven from the upstream end of the FFS to the target plane. 


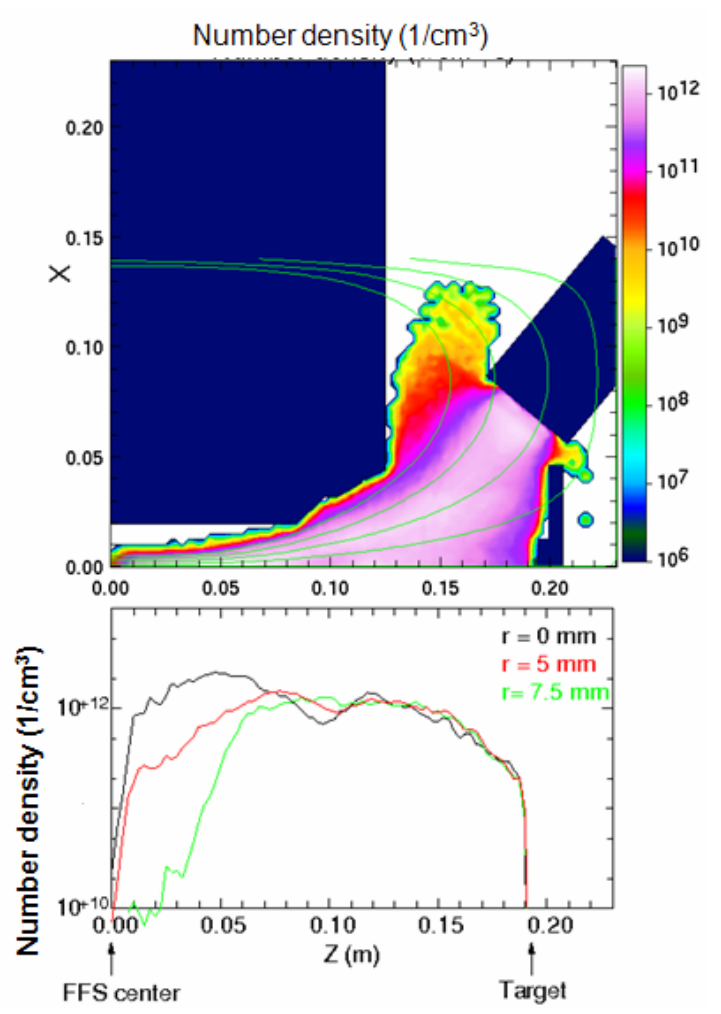

Fig. 7. 3-D electrostatic PIC simulation of the four-CAP plasma density when the FFS has B = $8 \mathrm{~T}$.

\subsection{Diagnostic improvements}

An improved Fast Faraday Cup has been installed in the target chamber. The electronic response still allows for $\sim$ nanosecond resolution of the ion beam current. The new design enables variation of gaps between hole plates, and hole plate transparency, easier alignment of front hole plate to middle (bias) hole plate, and a metal enclosure for shielding from the background plasma and electronic noise. Each hole plate is patterned with a hexagonal, two-dimensional array of holes. In the current version, the front hole plate (held at ground potential) has 9-mil diameter holes spaced apart by 40 -mils, while the middle hole plate (suppressor grid, biased to $-75 \mathrm{~V}$ ) has 18 -mil diameter holes. The collector plate is biased to $+75 \mathrm{~V}$. A schematic of the Fast Faraday Cup is shown in Fig. 8 below. The net transparency of the two hole plates results in a reduction of the beam current reaching the collector grid by a factor of 44 . 

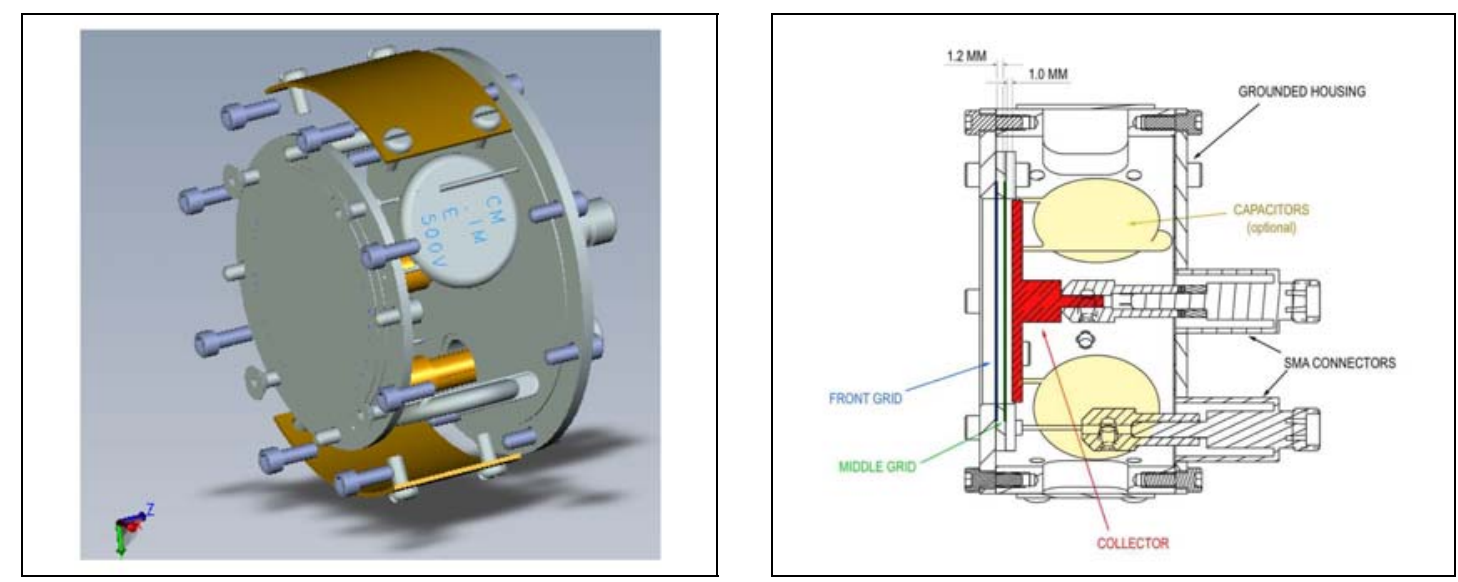

Fig. 8 : Fast Faraday Cup. Exploded view (left) and schematic view (right). Some of the installed side shielding is absent to display the interior.

\subsection{Temporal Beam Characterization}

The Faraday cup in the Diagnostic Box has been used to measure the beam current transported to the entrance of the IBM. This measurement is used in a cross-normalization with the beam signal measured in the Target Chamber with the Fast Faraday Cup on a later pulse to establish the beam current and power impinging on the target plane.

An example of the measured Faraday cup response to the beam current generated during a 330$\mathrm{kV}$ diode extraction is shown in Fig. 9. The linear fit to the flattop (green) shows the beam carrying $35.8 \mathrm{~mA}$ with a $0.7 \mathrm{~mA} / \mu \mathrm{sec}$ droop associated with the droop of the Marx voltage over this period. From this measure the beam is delivering $11.8 \mathrm{~mJ} / \mu \mathrm{sec}$ beam power over the flattop region.

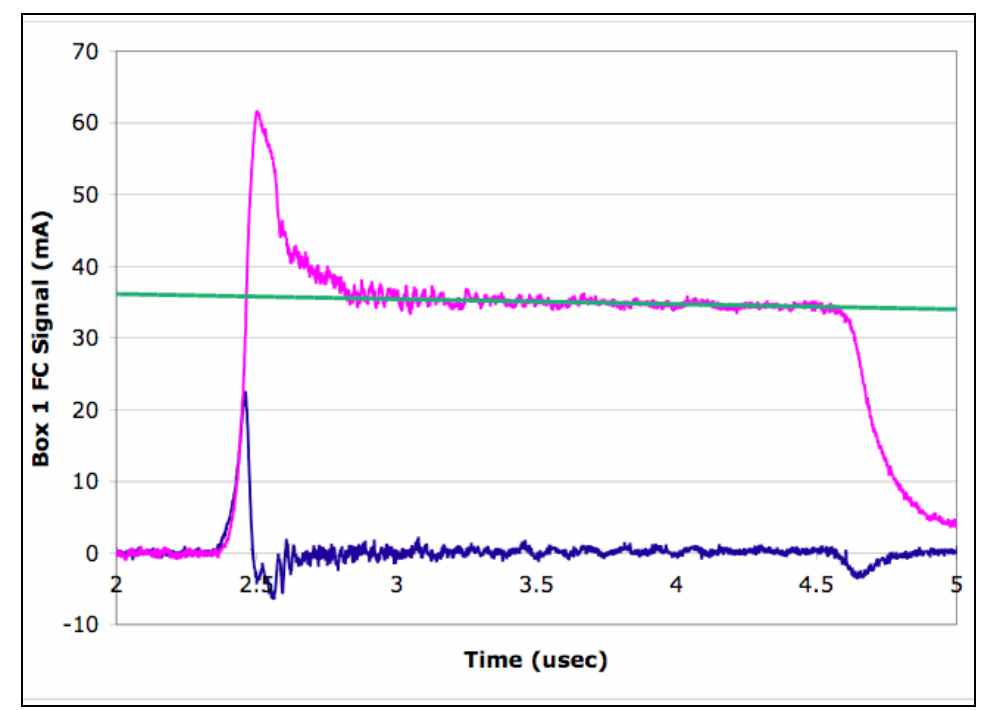

Fig. 9: Diagnostic Box Faraday cup beam current measurement. Shown is the collector plate signal (pink), the suppressor grid signal (blue), and a linear fit (green) to the collector signal flattop. 
The small change in beamline length due to the replacement of the high field Final Focus Solenoid required that the waveform for the IBM be retuned to provide optimum compression at the target plane position. This has been performed, with a relative difference less than $5 \%$ between the ideal and the applied waveforms over $\sim 200 \mathrm{~ns}$. The applied waveform, the ideal, and the voltage difference over 300 ns of the beam pulse are shown in Fig. 10.

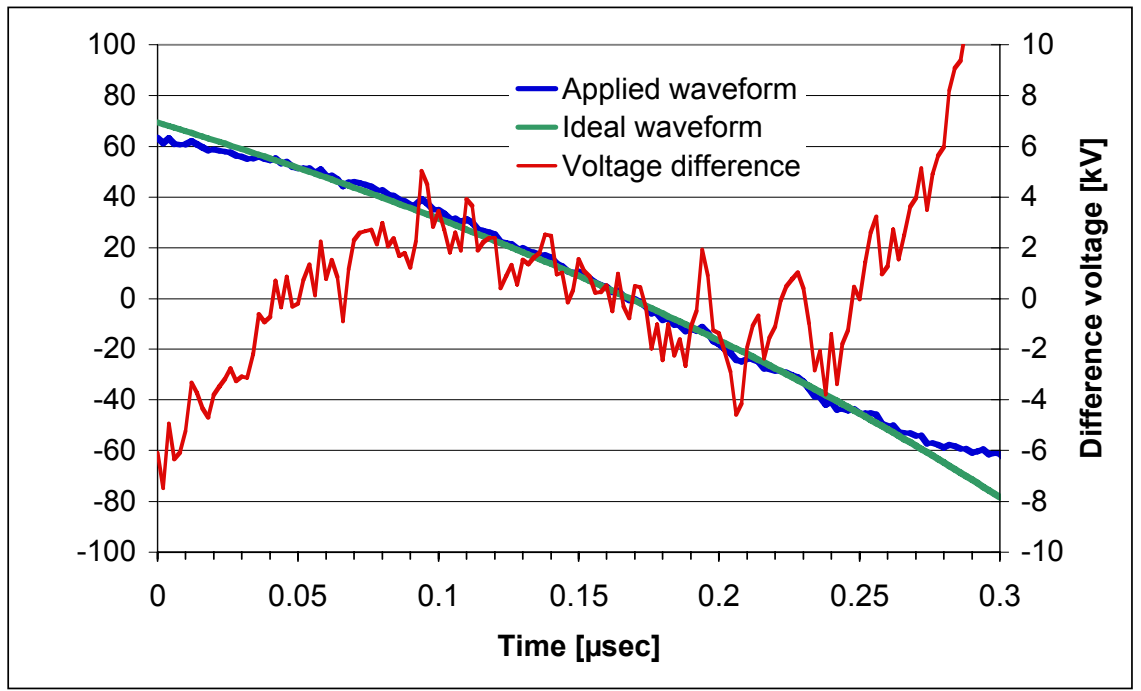

Fig. 10: IBM voltage waveform compared to the Ideal waveform.

The beam current profile arriving at the target plane is acquired by the Fast Faraday Cup and recorded on a Tektronix TDS 654C oscilloscope, and is shown Fig. 11 below. The beam arrival and uncompressed region of the pulse are clearly seen in the region from $3 \mu$ s to $\sim 4.5 \mu \mathrm{s}$, where the arrival time is measured with respect to the Marx trigger. The compressed pulse is clearly seen at $\sim 4.75 \mu$ s.

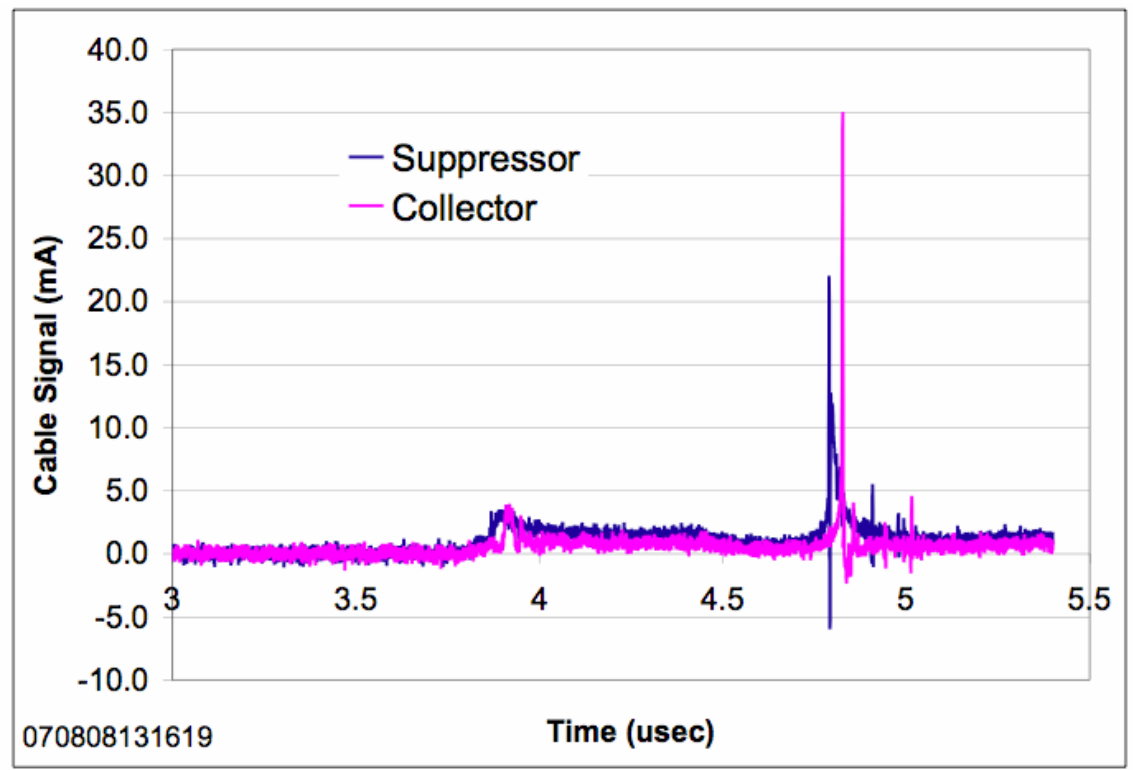

Fig. 11: Fast Faraday Cup signal of the uncompressed beam head and the compressed pulse region. 
The Fast Faraday Cup transparency factor of 44 can be used to derive the ion beam current hitting the front hole plate, and produces excellent agreement with the uncompressed current measurement obtained in the upstream Diagnostic Box when the beam is matched to the channel and is transported without scraping in the FEPS or Target Chamber.

In a well-tuned system, the beam compression reaches a maximum at the target plane. A fast (2.5 GSample/sec) acquisition oscilloscope (Tektronix TDS 654C) is matched to the Fast Faraday Cup to measure the distribution of current around the compression peak. For a $330 \mathrm{keV} / 35.8 \mathrm{~mA}$ beam, four measurements are overlaid and shown in Fig. 12.

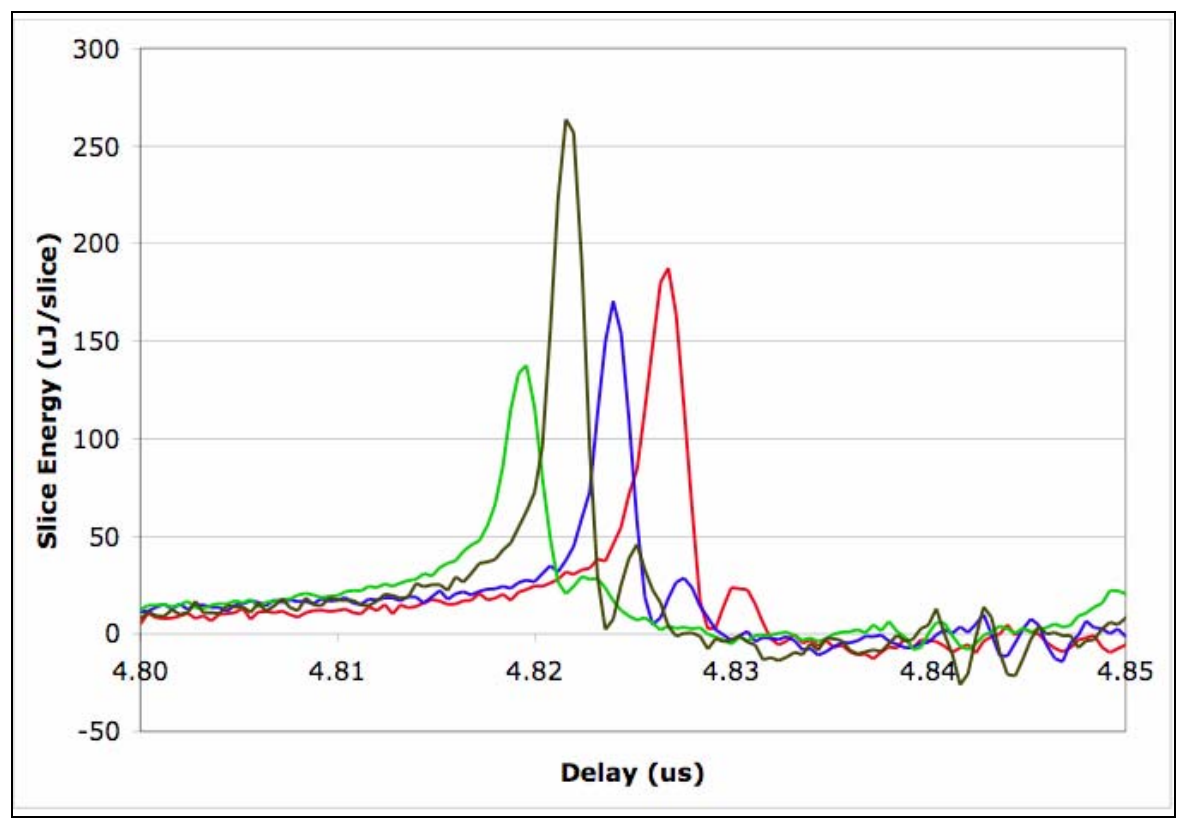

Fig. 12: Four compressed pulse profiles in a $330 \mathrm{keV} / 35.8 \mathrm{~mA}$ beam. The slice bin duration is 400 ps.

The parameters of these compressed pulses are shown in Table 1.

Table 1 : Compressed pulse parameters.

\begin{tabular}{|r|c|c|c|c|}
\hline Pulse & 1 (red) & 2 (blue) & 3 (green) & 4 (brown) \\
\hline Compressed pulse energy (mJ) & $\mathbf{1 . 8 3}$ & $\mathbf{1 . 8 4}$ & $\mathbf{1 . 9 9}$ & $\mathbf{2 . 0 0}$ \\
\hline Compressed pulse duration, 10\%-10\% $(\mathrm{ns})$ & $\mathbf{1 4 . 8}$ & $\mathbf{2 0 . 8}$ & $\mathbf{2 3 . 6}$ & $\mathbf{1 0 . 8}$ \\
\hline Compressed pulse duration, FWHM (ns) & $\mathbf{2 . 4 0}$ & $\mathbf{2 . 4 0}$ & $\mathbf{2 . 8 0}$ & $\mathbf{2 . 4 0}$ \\
\hline
\end{tabular}

The compressed pulse energy is found to be $1.915 \mathrm{~mJ} \pm .093 \mathrm{~mJ}$. The RMS amplitude jitter is then approximately $5 \%$. The timing jitter derived from a 10 -pulse set of measurements is shown in Table 2. 
Table 2 : Compressed pulse timing jitter measurements.

\begin{tabular}{|c|c|c|}
\hline $\begin{array}{c}\text { RMS Variation in Current } \\
\text { Peak arrival time (ns) }\end{array}$ & Average FWHM (ns) & $\begin{array}{c}\text { RMS Variation in FWHM } \\
\text { (ns) }\end{array}$ \\
\hline $\mathbf{5 . 3}$ & $\mathbf{2 . 3 7}$ & $\mathbf{1 . 1 3}$ \\
\hline
\end{tabular}

The variation in compressed pulse energy and the timing jitter can, in part, be attributed to voltage fluctuations of 5\% and 10-ns fluctuations induced by the IBM pulse.

\subsection{Spatial Beam Characterization}

The transverse (spatial) distribution of the beam is measured by allowing the beam to strike a 100-micron thick alumina scintillator, and then detecting the beam induced light emission with an image-intensified gated-MCP camera. The optical system produced a resolution of 10-17 pixels $/ \mathrm{mm}$ at the image plane. Image acquisition and processing software are then used to collect and analyze the time-gated images of the beam.

Optical measurements were performed with a scintillator placed at the target plane to observe the variation in beam spot size as the beam envelope matching conditions are varied. This is typically performed by changing the charging voltage in the fourth solenoid (S4) power supply, which linearly varies the peak field and adjusts the beam convergence angle as it enters the IBM and downstream neutralized plasma channels. The variation of the compressed and uncompressed spot radii (50\% integrated intensity measure) as the S4 charging voltage is changed is shown in Fig. 13. In this series of measurements, the uncompressed beam is represented by a 2300 -ns beam pulse without any voltage swing from the IBM. The compressed beam is represented by a 500 -ns gated image in the otherwise 2300 -ns duration beam pulse with an applied IBM voltage swing. This 500-ns gate is a factor of 2-3 longer than the IBM pulse, but accounts for slippage in the beam arrival time as the beam energy (and hence velocity) is also varied from $300-350 \mathrm{keV}$. The $50 \%$ integrated beam intensity spot radius is used since it provides a more robust measure than an RMS or edge radius measure.

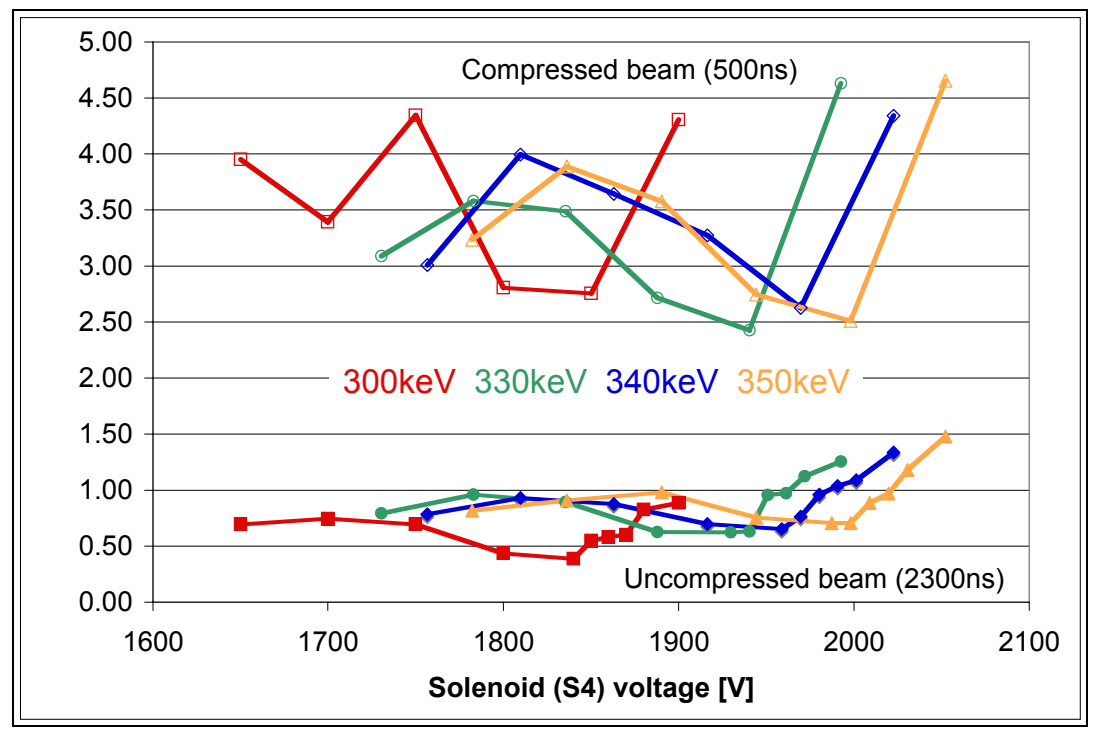

Fig. 13 : Compressed and uncompressed beam 50\% radius. 
The beam radii of the compressed and uncompressed beams show similar patterns as the solenoid charging voltage changes. This reflects the scaling properties of the beam envelope as the beam energy and solenoid field vary simultaneously. The uncompressed $300 \mathrm{keV}$ beam shows a minimum spot radius for $\mathrm{S} 4=1850 \mathrm{~V}$. The $350 \mathrm{keV}$ shows the same minimum at $\mathrm{S} 4=(1850 \mathrm{~V})$ $*(350 / 300)^{1 / 2}=1998 \mathrm{~V}$. Using this similarity argument, we can say that the minimum occurs for all beam energies when $\{\mathrm{S} 4\}=1850 \mathrm{~V}$, where it is agreed that we are referencing the solenoid field for $300 \mathrm{keV}$ beams.

The large (factor of 4) difference between the compressed and uncompressed beam spot radii can be shown to arise from the large chromatic aberrations found in the compressed pulse induced by the voltage swing from the IBM as well as the large integration time for the compressed pulse image acquisition.

Two examples of the beam spot for an uncompressed $340 \mathrm{keV} / 36 \mathrm{~mA}$ beam are shown in Fig. 14. The peak fluence values are shown for $1.0-\mu$ s integration times.

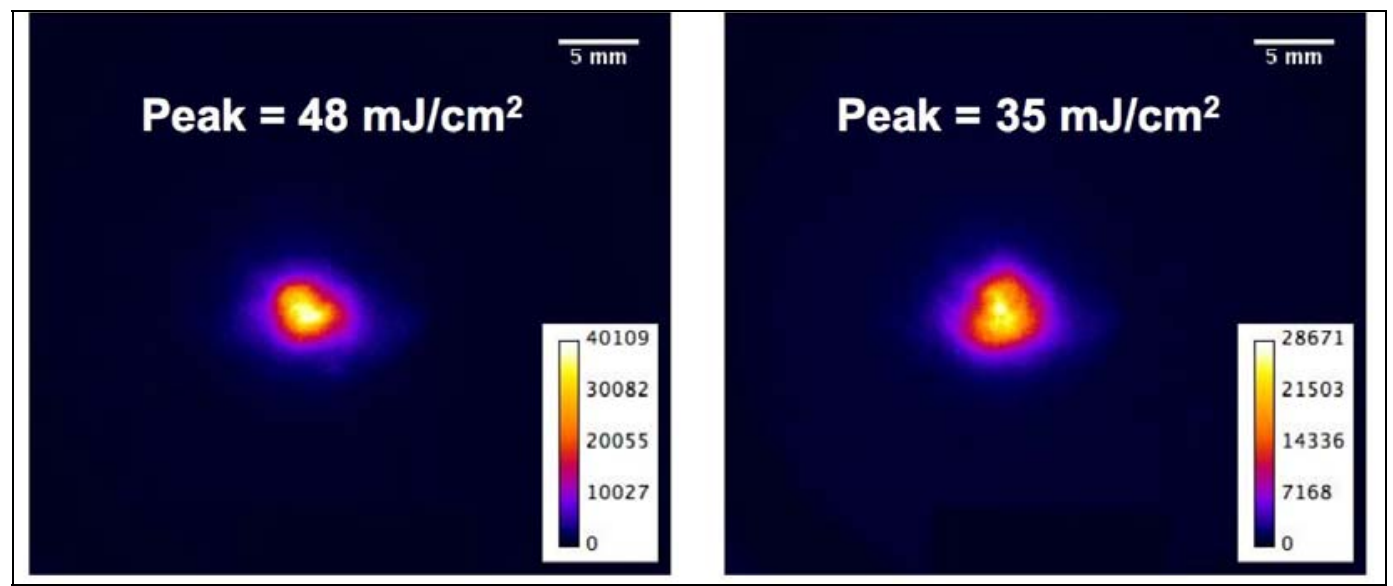

Fig. 14 : Fluence distribution in an uncompressed beam.

The transverse centroid jitter in the uncompressed beam poses a limiting factor to the amount of target heating one can obtain. Centroid measurements have been performed as the beam energy and S4 solenoid field are varied. The results are shown in Fig. 15. Clearly, the beam centroid lies within a 200-micron 'circle of confusion' that arise from variations in pulse energy and solenoid field strength. 


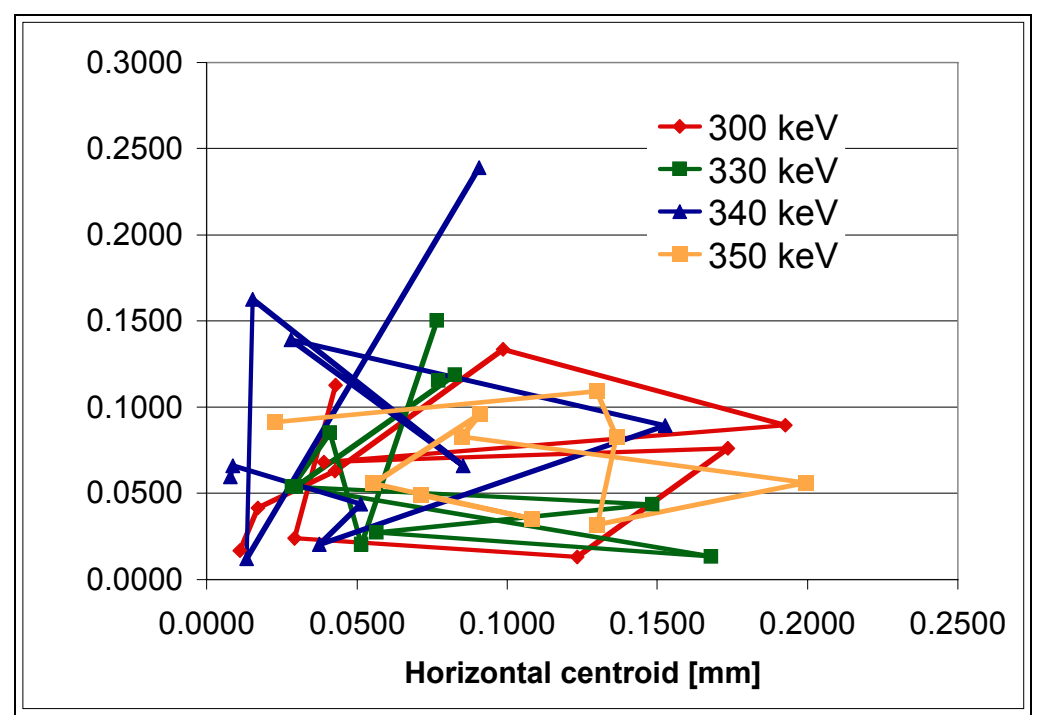

Fig. 15: RMS centroid jitter (mm) for the uncompressed beam

To perform a more careful analysis of the spatial distribution and variations of the compressed portion of the beam pulse, a much smaller image gate (10 ns) is chosen. For a particular beam energy, the arrival time of the compressed pulse is constant (to within the measured jitter). For our $330 \mathrm{keV}$ beam, the average pulse width of $2.37 \mathrm{~ns}$ has an arrival time jitter of $5.3 \mathrm{~ns}$. The spot size variation as the S4 solenoid field strength is changed is shown in Fig. 16.

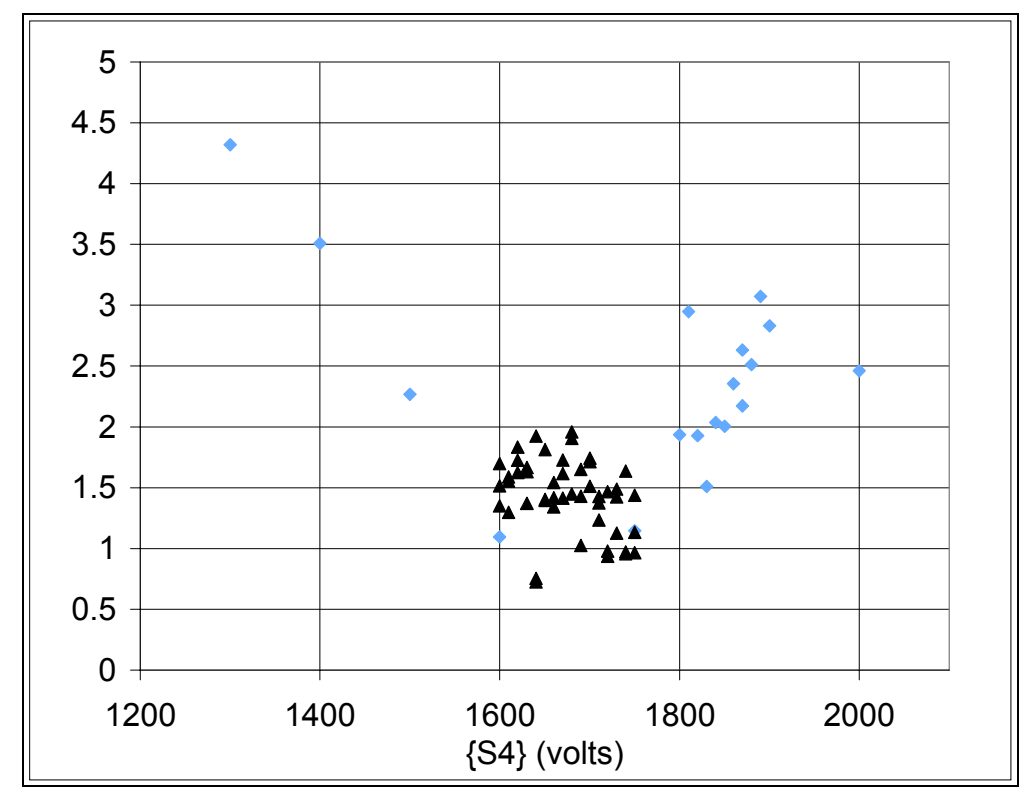

Fig. 16 : Variation of compressed beam $50 \%$ spot radius with solenoid (S4) charge voltage for a $330 \mathrm{keV}$ beam.

From this Figure we see that the optimum tune of $\{\mathrm{S} 4\}=1700 \mathrm{~V}$ varies from that of the compressed portion of the beam, where the optimum tune is $\{\mathrm{S} 4\}=1850 \mathrm{~V}$. A joint optimization may be required based upon another figure of merit, if this condition is left uncorrected. Alternatively, correcting the chromatic aberrations residing in the compressed portion of the 
pulse may restore the optimum tune and spot radius for both compressed and uncompressed portion of the beam pulse.

For a tune that optimizes the compressed beam fluence, the variation of spot radius with arrival time is shown in Fig 17. Here the changes to the spot radius and transverse distribution are evident as we scan across the compression peak.

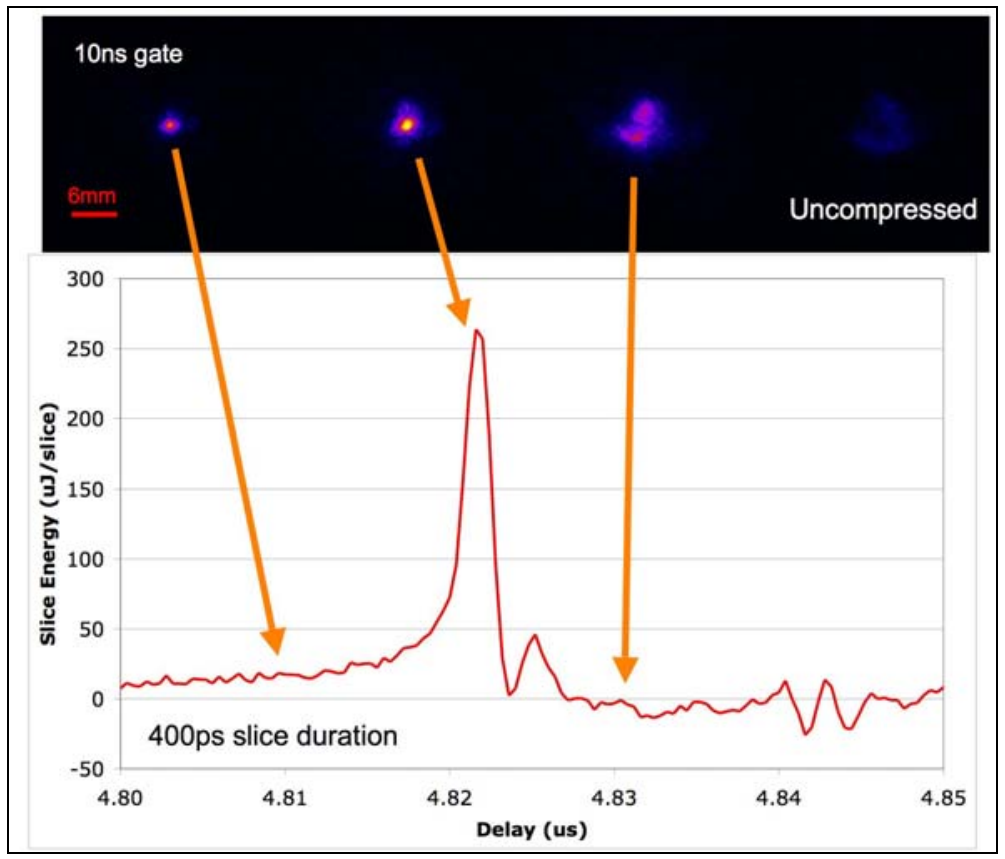

Fig. 17: Beam distribution and slice energy over the compressed region of the pulse.

Measurements of the compressed beam fluence were performed for both 10-ns and 2-ns image gates, centered on the compressed pulse peak for a $330 \mathrm{keV} / 35.8 \mathrm{~mA}$ beam. Lineouts across the image centers provide a 1D measure of the fluence field of the beam at the target plane, shown in Fig. 18. In these two cases, the peak fluences were found to be in the range $0.95-1.2 \mathrm{~mJ} / \mathrm{cm}^{2}$ integrated over $1 \mathrm{~ns}$. 


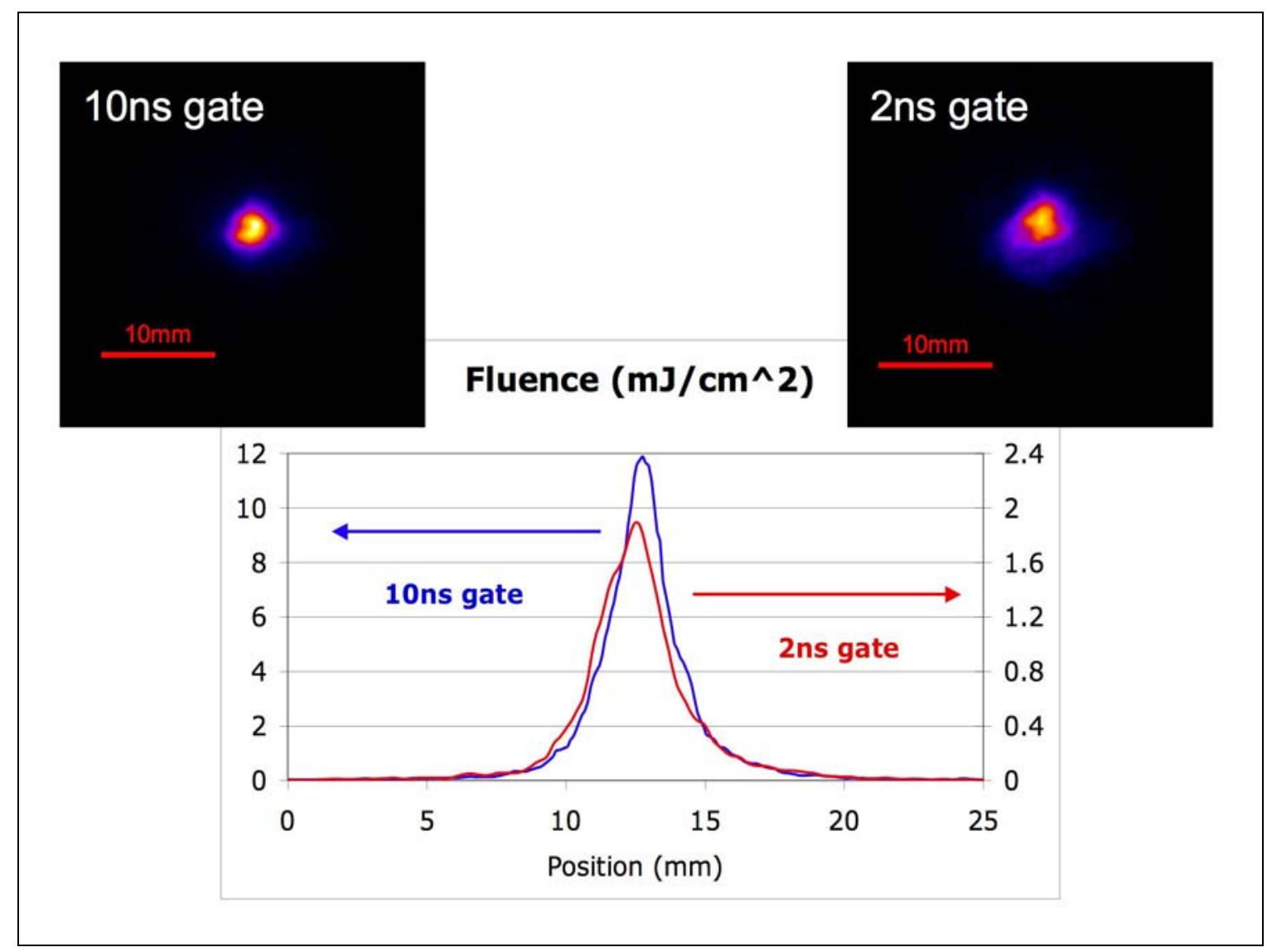

Fig. 18: 1D fluence distribution for compressed pulses at the peak of compression. 


\section{TARGET EXPERIMENTS}

\subsection{INITIAL TARGETS AND MODELING}

WDM targets in hand include free-standing foils (e.g. 350-nm Al, 150-nm Au) and thin films deposited on a sapphire substrate (350-nm Al, 150-nm Au and 150-nm W). These targets have a thickness comparable to the range of the $300-\mathrm{keV} \mathrm{K}^{+}$beam from NDCX, so that the energy deposition distribution is nearly uniform. Simulations using HYDRA of the temperature distribution in typical targets were performed considering that the uncompressed portion of the NDCX pulse delivers a fluence of $35 \mathrm{~mJ} / \mathrm{cm}^{2}$ every microsecond; it was also assumed that a compressed 350-keV K $\mathrm{K}^{+}$beam of 2-A, 2-ns, and 1-mm diameter, can be delivered to the target, which corresponds to an energy density of $0.1 \mathrm{~J} / \mathrm{cm}^{2}$. (The currently measured energy density is $\sim 4 \mathrm{~mJ} / \mathrm{cm}^{2}$, but much optimization, including insertion of a focusing cone and time dependent focusing, remains to be done.) The calculations show that the target heats up past the melting point without appreciable hydro motion expanding only a few nanometers. In this calculations tin (Sn) was used in place of gold in order to be able to use the QEOS equation of state tables. In general the temperature of a target increases as the target atomic mass increases, so that the gold and tungsten targets are expected to reach a higher temperature than indicated here for tin and aluminum.

NDCX produces a compressed pulse ( $\sim 2$ ns duration) above a broad uncompressed pulse. For high-pressure experiments the compressed pulse is necessary, but interesting experiments can be done with the uncompressed pulse. This uncompressed pulse potentially contains much larger energy. We estimate that it is possible to deposit $>10$ kilojoules $/ \mathrm{cm}^{3}$ in a 0.3 micron layer during a $10-\mu$ s ion beam pulse.

The special conditions of NDCX favor an interesting class of experiments likely to provide important information about hot liquid metals and the liquid-vapor two-phase region.

Because the heating rate is slow compared to the time required for thermal expansion, the heated material will expand during heating and remain at low pressure. It will melt and rise up the highdensity side of the two-phase boundary. With sufficient energy it can go well above the nominal boiling point and can even approach the critical point. As the temperature rises the surface evaporation rate increases. Apart from evaporation, the liquid metal will retain a sharp surface sustained by surface tension. We expect during the prepulse the foil temperature will be determined by an equilibrium between heating by the beam and cooling due to evaporation and electron emission. During the compressed pulse, a higher temperature can be reached because of the higher heating rate.

A simple model of the heating process assumes that (neglecting cooling from thermal electron emission, which is expected to be small at this temperature) the target will melt and surfaceevaporate until it reaches the flux equilibrium condition where the energy evaporation rate flux $\left(\mathrm{Lnv}_{\mathrm{t}} \exp (-\mathrm{L} / \mathrm{kT})\right)$ equals the beam energy flux. Here $\mathrm{L}$ is the latent heat of vaporization per atom, $\mathrm{kT}$ is the target temperature, $\mathrm{n}$ is the number density of the target, and $\mathrm{v}_{\mathrm{t}}$ is the thermal velocity. For example, for the uncompressed part of the NDCX pulse, we have an estimated fluence of $35 \mathrm{~mJ} / \mathrm{cm}^{2} / \mu \mathrm{s}$; the $150-\mathrm{nm}$ gold foil target reaches the flux equilibrium condition at a 
temperature of $\sim 3600 \mathrm{~K}$ in about $5 \mu \mathrm{s}$. At $3600 \mathrm{~K}$ it would take about $15 \mu$ s to vaporize the entire target. During the compressed pulse the target equilibrium is reached at a higher temperature, and for a compressed pulse of sufficient energy significantly higher temperatures can be obtained.

An alternative hypothesis of the gold foil heating process is that the target will melt and volumetrically form bubbles near the nominal boiling point. This would clamp the temperature at a lower value than calculated above assuming surface vaporization and would increase the total vaporization rate because of the increased surface area of the bubbles.

To date the critical points are not known for metals such as aluminum, iron, tantalum, molybdenum, and tungsten. (Estimates in the literature differ by factors of two.) In addition, there are large uncertainties in the liquid vapor boundary at high temperature. Experiments on NDCX can greatly reduce this uncertainty. The foil temperature can be measured by pyrometry, a technique which can be calibrated by using high-melting samples as calibration standards. The evaporation rate can be determined by optical laser probe reflection and by monitoring beam transmission through the foil as it evaporates. This information will greatly improve EOS models for matter in the WDM range of temperatures and densities.

\subsection{TARGET DIAGNOSTICS}

The primary target diagnostics are a fast pyrometer for temperature measurements, streak camera, spectrometer, VISAR and an optical camera for viewing the target from the rear. The diagnostics and initial test data, including the light collection system, and fiber optics lines and fiber optics feedthroughs are described in Ref. 1.

\subsection{TARGET EXPERIMENTS}

The target chamber and target diagnostics are now operational. We have performed three beamtarget experiments to date; each experiment has developed and improved our capability to perform and diagnose these experiments.

In our first beam-target interaction experiment, the $350-\mathrm{keV}, 37-\mathrm{mA}, 3-\mu \mathrm{s}(39 \mathrm{~mJ})$ beam pulse was directed onto a self-supporting $150 \mathrm{~nm}$ gold foil. The purpose of this test was to characterize the effect of the beam on the target foil. For this initial test the target diagnostic was the gated PIMAX camera viewing the foil from downstream. An image of the beam on the scintillator before the target experiment is shown in Fig. 19. Also shown in the image are calibration marks on the surface of the scintillator. We obtained images of the target before, during, and after the beam pulse (Fig. 20). The center image shows light emitted from the target foil during the beam pulse. Figure 21 shows a picture of the target foil after the beam pulse on a scintillator, showing the hole created in the foil by the beam. The hole is smaller than the beam 
cross section showing that the target exceeded the melting point near the peak of the beam distribution.

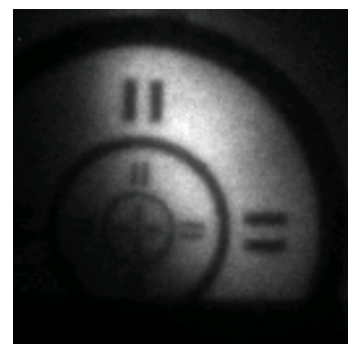

Figure 19. Profile of the beam on a scintillator placed at the target location. The distance between the two marks outside the innermost circle is $1 \mathrm{~mm}$.

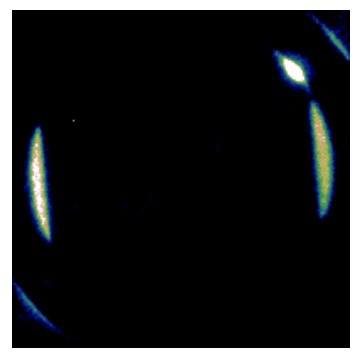

before

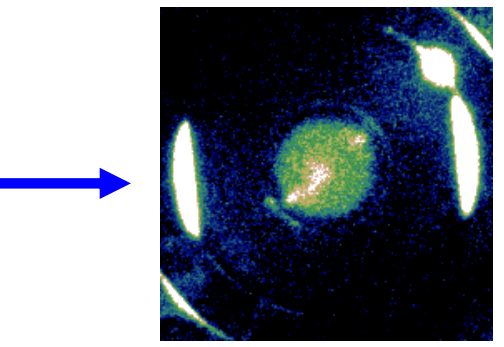

During pulse
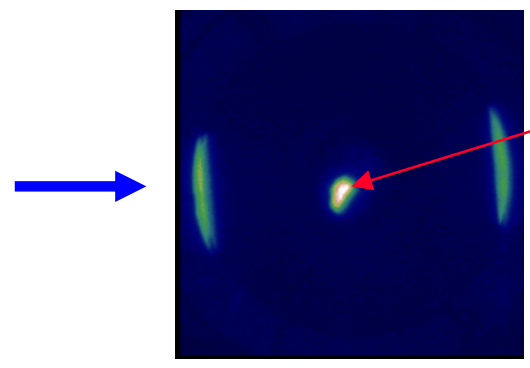

after
Light from

ion source

through

hole

Figure 20. Images of the target foil before, during, and after the NDCX beam pulse.

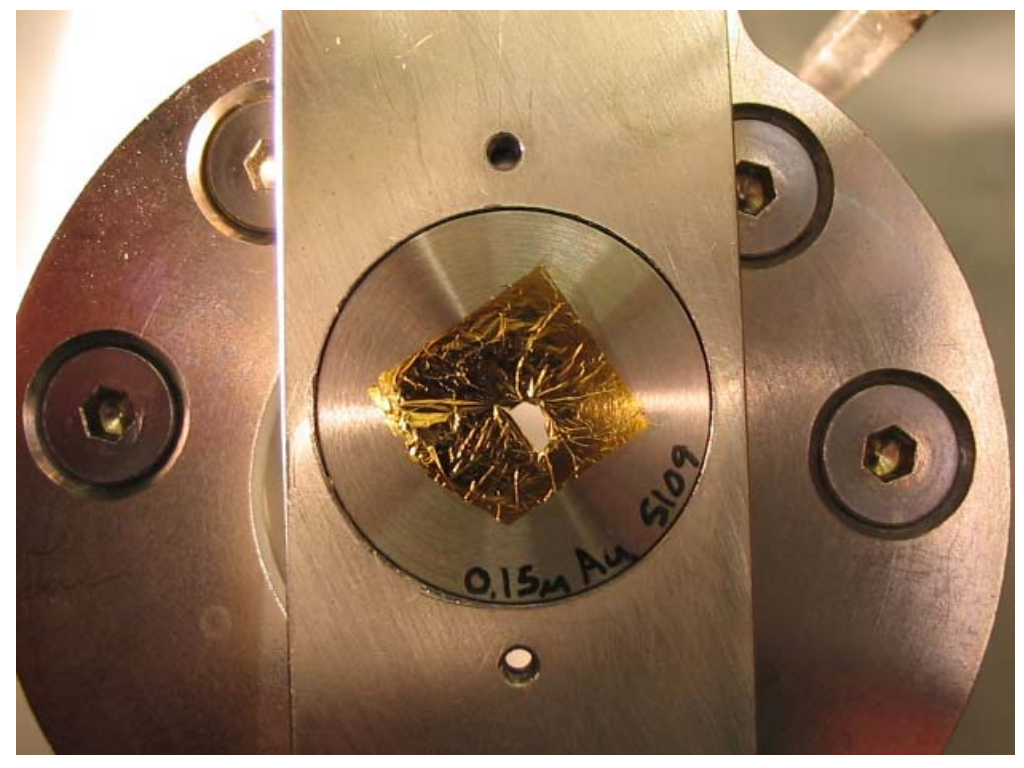

Figure 21. Picture of the gold target foil after interaction with the NDCX beam. The outer diameter of the foil holder ring is $17 \mathrm{~mm}$ and the inner diameter is $5 \mathrm{~mm}$. The hole is approximately 2.3 by $1.1 \mathrm{~mm}$. 
The second target experiment was viewed for the first time using the new light collection optics. In this experiment the target was simultaneously viewed with the fast optical pyrometer through a 400-micron diameter fiber and imaged through a fiber optic light guide onto a PIMAX gated ICCD camera. This experiment tested procedures for alignment between the location of the beam pulse at the target and the imaging location of the light collection system. Figure 6 shows a (false color) image of the gold target during the $10-\mu$ s beam pulse showing light emitted from the target as imaged from the rear through the light guide. The white circle indicates the spot probed by the pyrometer as indicated by a calibration laser sent through the optical probing fiber and the light collection system. It shows that the probed spot was not at the peak of the beam distribution and as a result the target temperature at the probing spot was too low to measure (significantly less than $2000 \mathrm{~K}$ ).

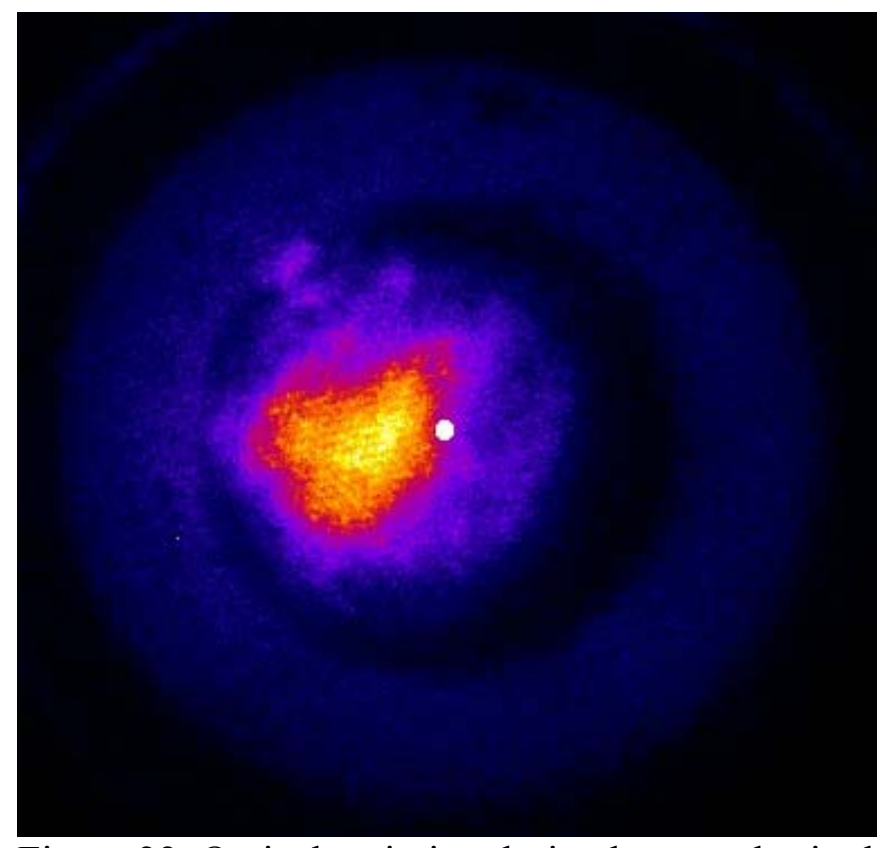

Figure 22. Optical emission during beam pulse in the second experiment on the gold target foil (10-mm diameter). The location of the spot probed by the optical pyrometer in the second experiment (shown) was not at the center of the beam. For the third experiment (not shown), the beam and probed spot coincided.

The third beam-target experiment was performed after improving alignment and calibration procedures in light of the result of previous test. In the experiment we focused a $>10 \mu \mathrm{s}$ NDCX beam pulse onto a free-standing 150-nm gold foil target. The location of the probed spot was moved to properly coincide with the location of the peak of the beam pulse to within measurement error. The compressed portion of the pulse arrived at the target about $8 \mu$ s into the pulse. Light emitted from the target was directed by the light collection system to the fast optical pyrometer and a fast gated camera. The beam prepulse heated the target to a temperature high enough to measure with our optical pyrometer, and the subsequent compressed pulse added a short burst of ion deposition energy in a few ns. This is the first measurement of target temperature in a beam-target interaction experiment on NDCX. Useful signal appeared on all three active pyrometer channels (Fig. 23). Preliminary analysis of the data shows that the brightness temperature (which assumes black body emission) peaked at about $2600 \mathrm{~K}$ (Fig. 24). 
The corresponding true target temperature is $>2600 \mathrm{~K}$ and can be estimated from the data. Further analysis of the time evolution of the measured temperature is underway.

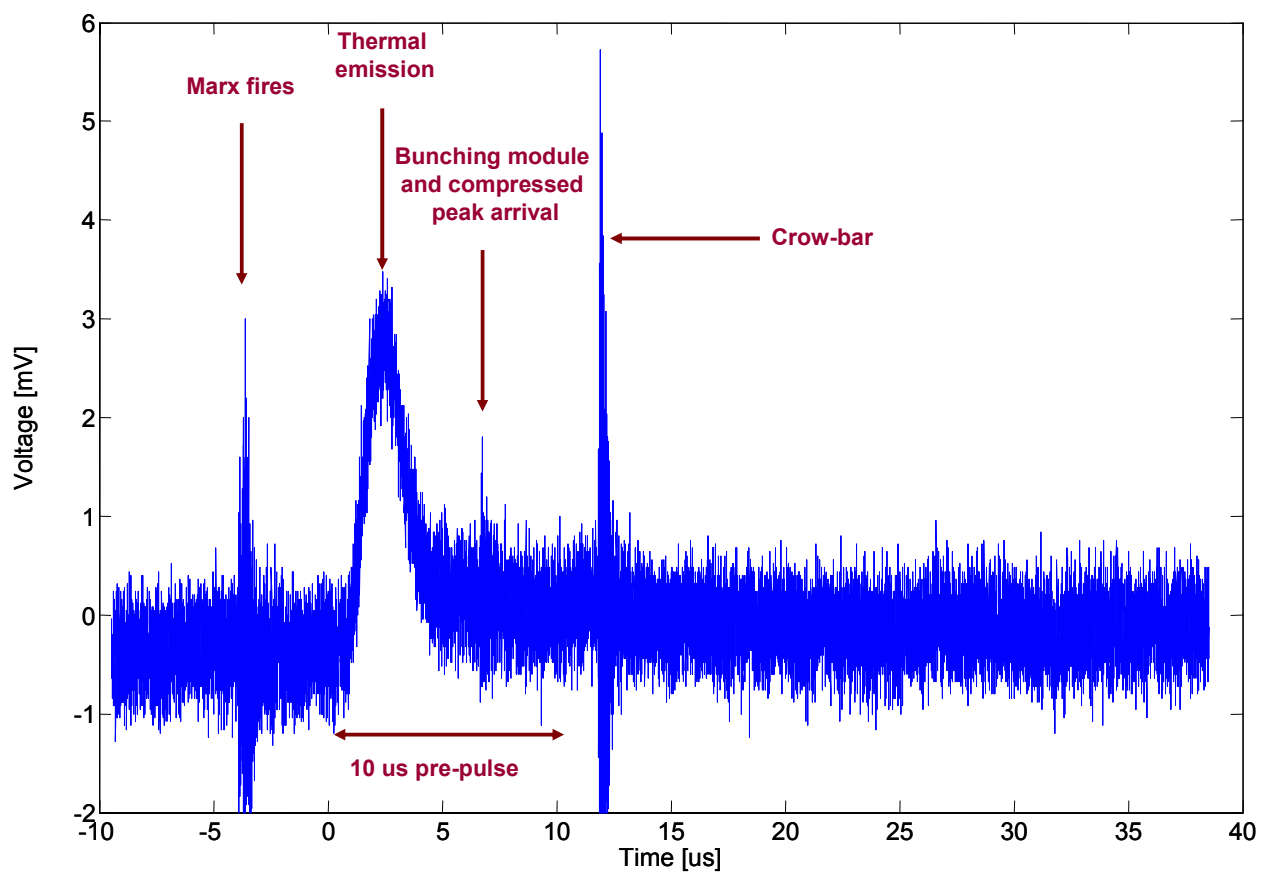

Figure 23. Output of the pyrometer detector measuring thermal emission from the target at a wavelength of $1000 \mathrm{~nm}$. Timing fiducials including noise bursts from the Marx bank, crowbar, and bunching module are indicated on the trace.

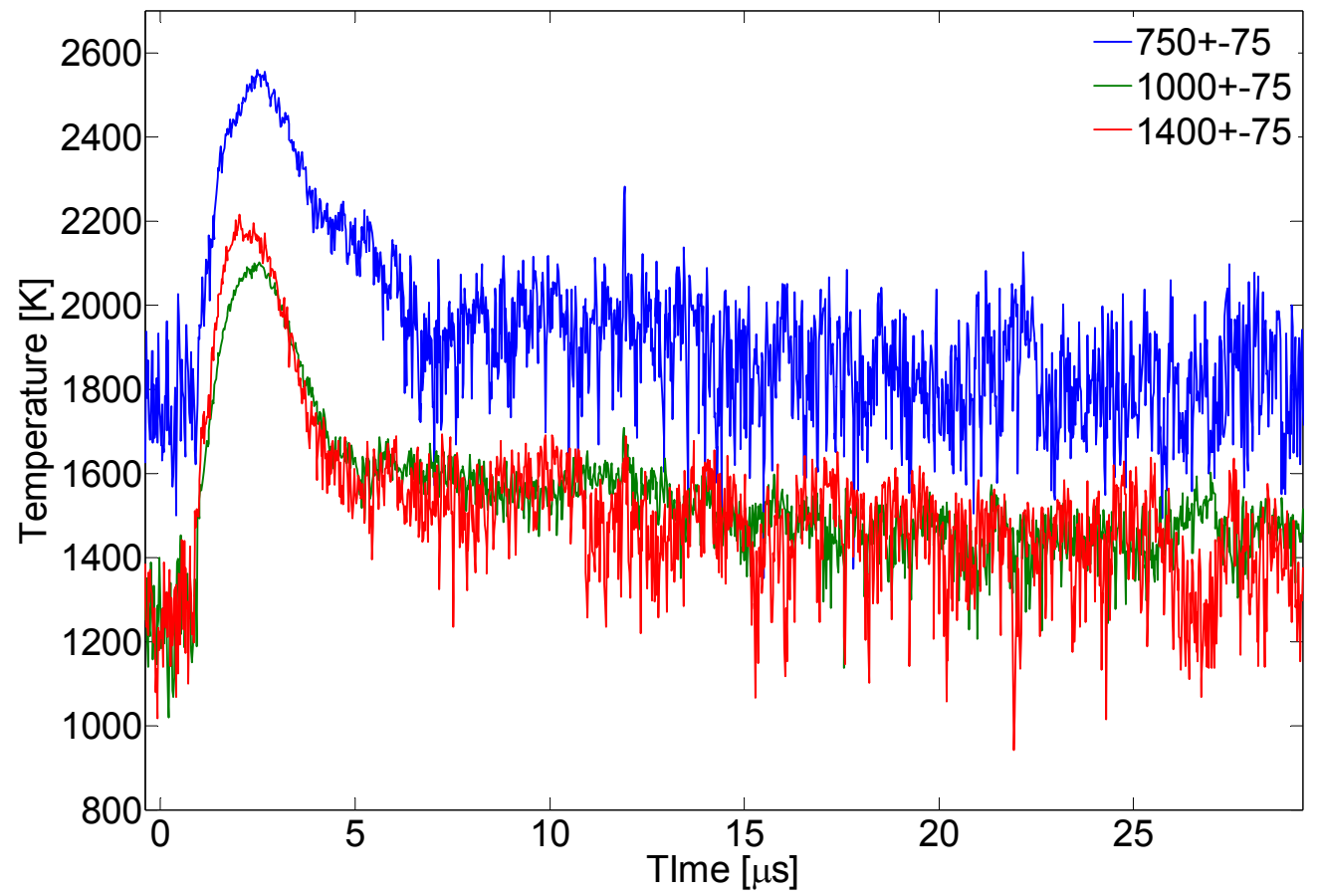

Figure 24. Brightness temperature at the three wavelengths monitored by the pyrometer. 


\section{CONE EXPERIMENT}

We are investigating the performance of a gold cone in concentrating the beam immediately upstream of the target. The cone is based on the reflection properties of the solid gold wall at grazing incidence. The particle reflection coefficient is shown in Fig. 25 for $\mathrm{K}^{+}$ion beam on a gold wall as calculated using TRIM. The energy reflection coefficient of the reflected ions is comparable to the particle reflection coefficient. Two cones have been tested, one which is a simple cone with straight walls at $140 \mathrm{mrad}$ angle of incidence, and the second a cone with a parabolic cone wall shape, with approximately $160 \mathrm{mrad}$ angle of incidence at the entrance and a 40-mrad angle of incidence at the exit plane. The second generation cone in its alignment fixture is shown in Fig. 26. The cone was characterized by passing a laser through the cone. The pattern of reflected light is shown in Fig. 27. It shows both the laser light that passes directly through the cone and a ring of light reflected from the inner cone wall. The degree of symmetry of the reflected light is an indication of the imperfection in construction of the cone. Both cones are approximately $1-\mathrm{cm}$ tall. Preliminary data from recent beam tests is summarized in Figs. 28 and 29. The results to date are encouraging and indicate that the enhancement is strong enough to continue investigation into the performance of the cone because of its potential to double beam intensity on target. Other advantages of the cone include the large amount of neutralizing secondary electrons expected from the grazing incidence at the cone walls, and the shielding of the target from the edges of the beam pulse. An alternative shield is a pinhole which has also been tested. However there is no enhancement of beam intensity with a pinhole.

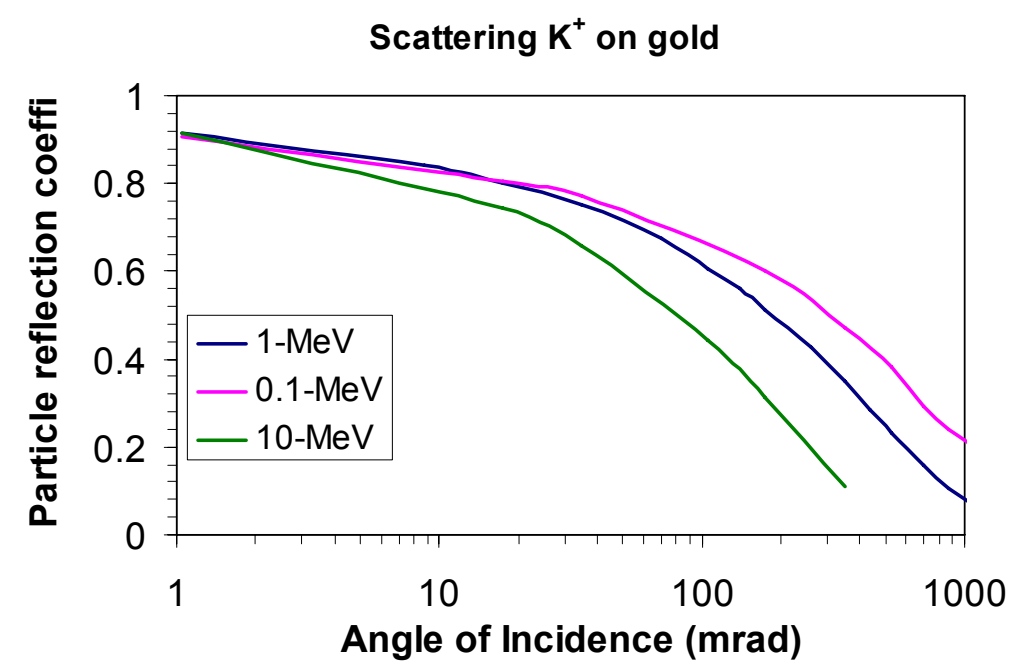

Figure 25. Particle reflection coefficient for $\mathrm{K}+$ ions on gold as a function of angle of incidence for three beam energies $(0.1,1$, and $10-\mathrm{MeV})$. 


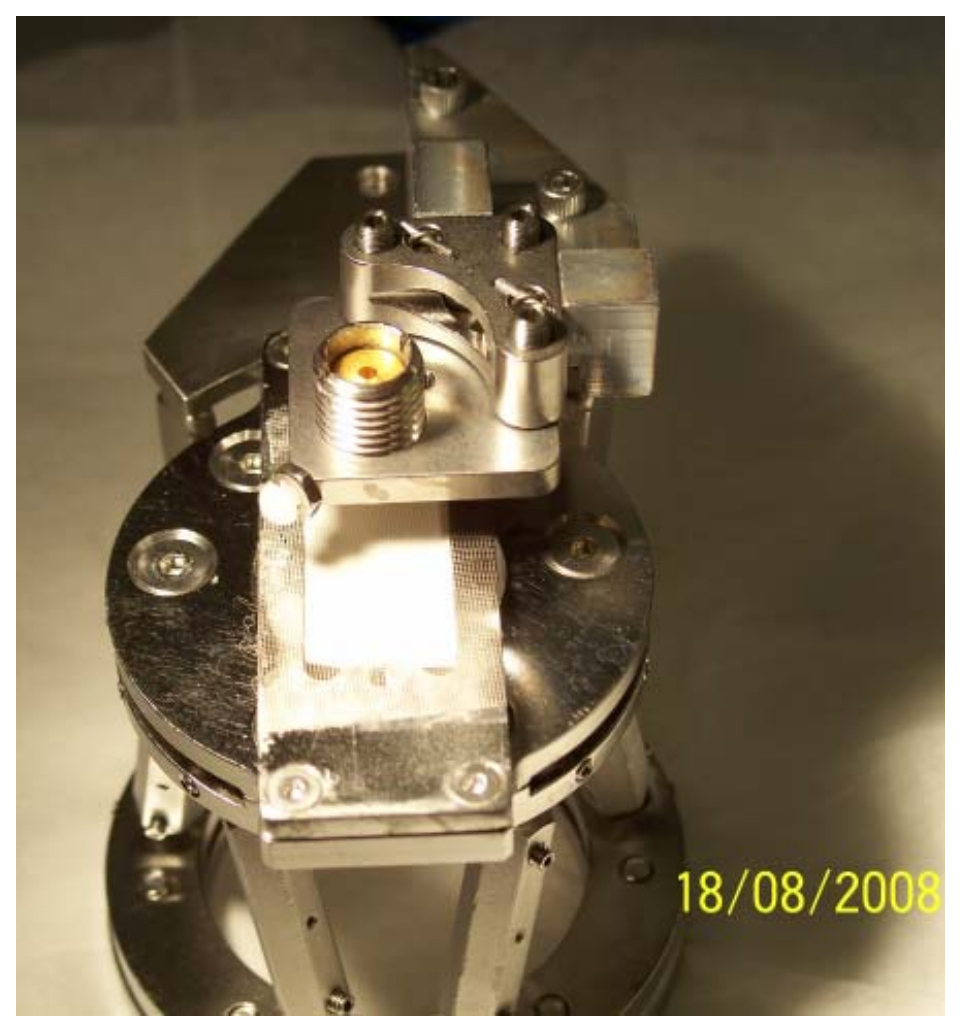

Figure 26. Gold cone with alignment fixture for test of concentration effect of cone using scintillator.

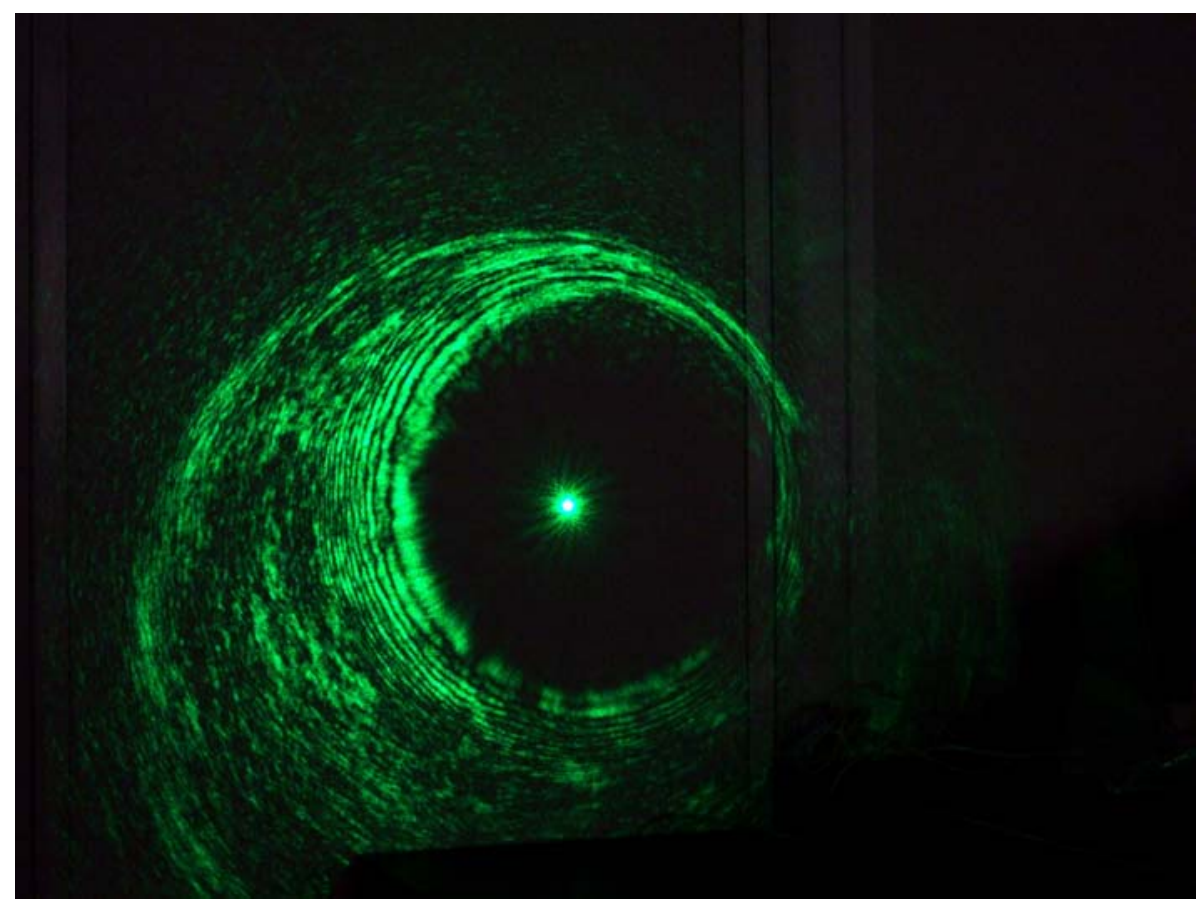

Figure 27. Laser light reflected on cone wall and projected $1.6 \mathrm{~m}$ shows light directly passing through the cone (center) and light reflected from the inner wall of the cone (ring). 


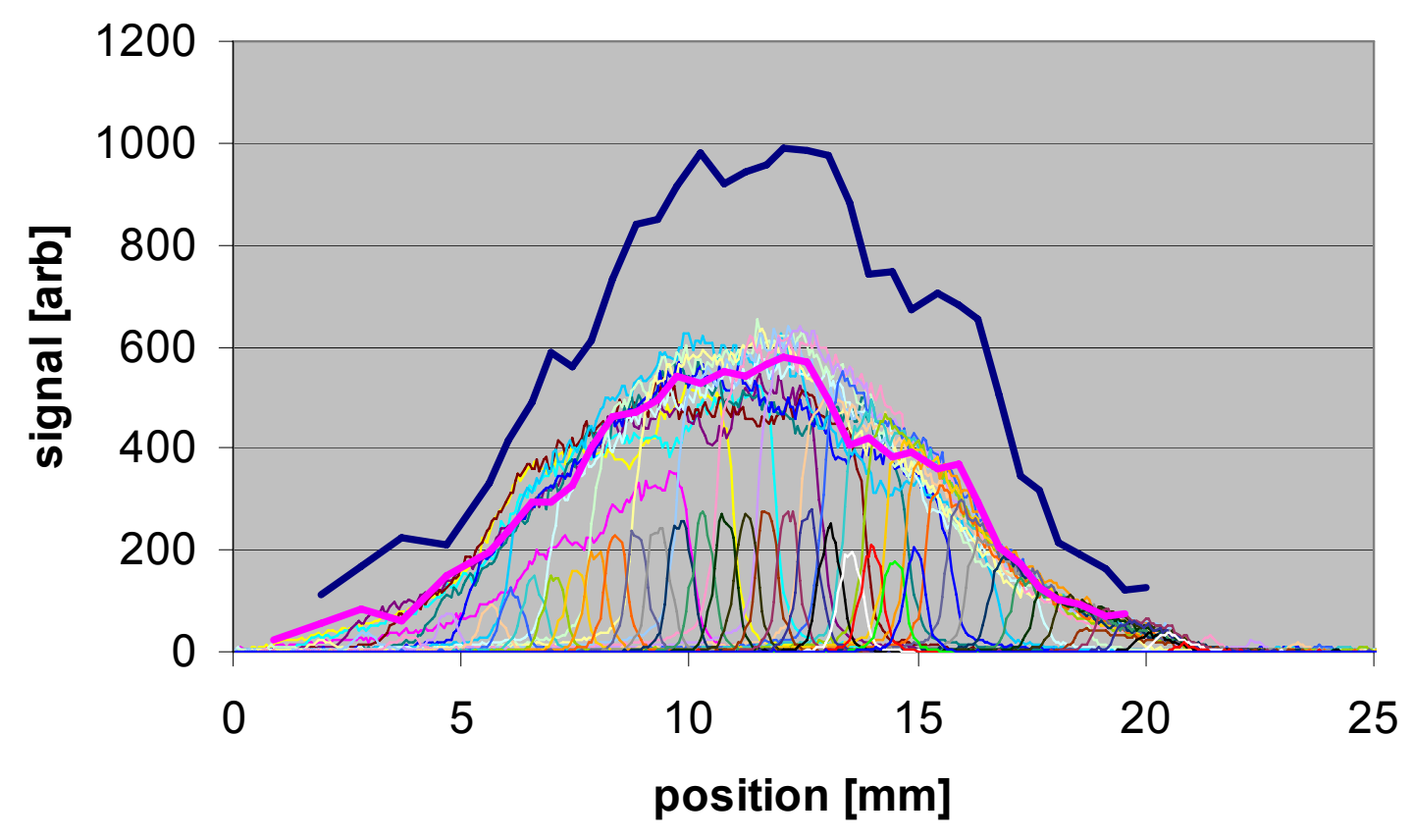

Figure 28. Preliminary result of a scan of the cone through the beam profile shows a beam intensity enhancement in the cone of a factor $\sim 1.8$ comparing the beam signal through the cone (blue) compared with the signal in the absence of the cone (light traces).

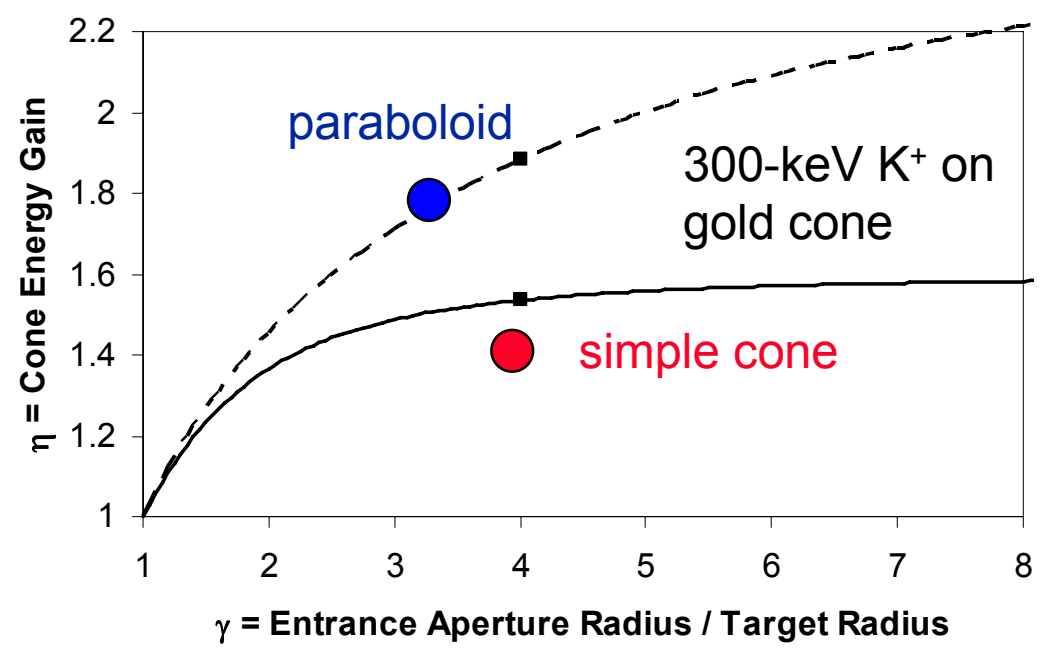

Figure 29. Summary of experimental data points and model predictions from TRIM (for a single reflection) of the two cone geometries tested. 


\section{PLANS FOR FUTURE WORK}

The initial experimental results are suggestive of potentially interesting physics. The rapid initial rise and subsequent decay of the target temperature during the beam pulse indicate changes in the balance of beam heating and target evaporative cooling, a behavior which may be affected by phenomena such as droplet formation and rapid changes in the optical properties of the hot target material. We plan to study the target behavior using target diagnostics already in place such as the fast pyrometer and streak camera, as well as by measuring ion beam transmission and optical transmission through the foil. Measurements with these diagnostic techniques can help determine the rate at which the target is breaking up into droplets and the rate at which its bulk optical properties are changing.

We plan to continue to perform beam-target experiments with increasingly sophisticated beam and target diagnostic capabilities. One of the areas of effort in the near term will be in improvements in target holder so that multiple experiments can be performed with a single target. The targets will be mounted behind a cone or hole plate which acts as a shield to mask the target from damage from the edge of the beam. Repositioning the target behind the shield will provide fresh target material and allow a number of beam-target experiments before replacing the target. Implementation of this upgrade will dramatically improve turnaround time between target experiments.

Further improvements to the target chamber are planned. For example, for a user facility a robotic target handling system will be required in order to be able to use up to 50 targets without interrupting the experimental program. Such an upgrade will require a vacuum load lock system and a custom designed, vacuum-rated robotic target handling system.

We will implement these and other improvements once we have gained sufficient experimental experience with the current setup. As future plans for target experiments will evolve we will improve and continually update the target chamber facility to advance the challenging experiments with ion-beam driven, isochorically heated warm dense matter.

\section{REFERENCES}

1. HIFS-VNL 3rd Quarter 2008 milestone report, June 12, 2008.

2. J.J. Barnard, et al., Accelerator and ion beam tradeoffs for studies of warm dense matter, Proc. 2005 Particle Accelerator Conference, p. 2568

3. P.K. Roy, at al., Neutralized drift compression experiments with a high intensity ion beam, NIM A 577 (2007) 223-230.

4. P.A. Seidl, et al., Plans for longitudinal and transverse neutralized beam compression experiments, and initial results from solenoid transport experiments, NIM A 577 (2007) 215-222. 
5. F.M. Bieniosek, J.J. Barnard, M.A. Leitner, R.M. More, P.K. Roy, Diagnostics for nearterm warm dense matter experiments, NIM A 577 (2007) 284-288.

6. P.A. Ni, et al., Diagnostics for warm dense matter experiments with intense ion beams, Proceedings HIF08, to be published in NIM A.

7. P.K. Roy et al., Space charge neutralizing plasma for beam drift-compression experiments, Proceedings HIF08, to be published in NIM A. 